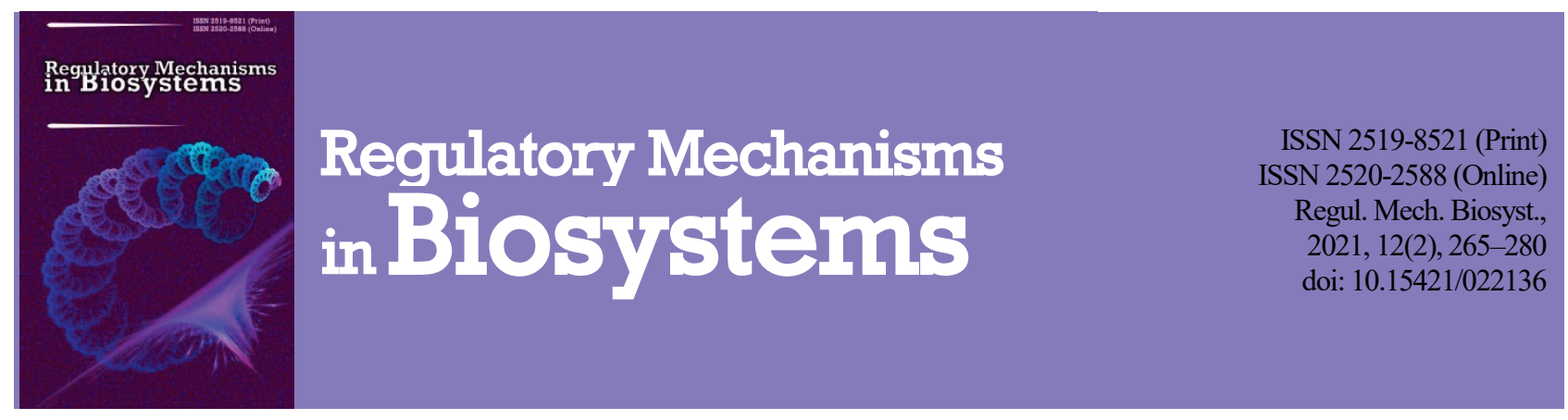

\title{
Anti-tumour drugs of marine origin currently at various stages of clinical trials (review)
}

\author{
E. A. Bocharova*, N. I. Kopytina*, E. E. Slynko*,**,*** \\ *A. O. Kovalevsky Institute of Biology of the Southern Seas of RAS, Moscow, Russia \\ **Papanin Institute for Biology of Inland Waters Russian Academy of Sciences, Borok, Russia \\ ***Yaroslavl State Agricultural Academy, Yaroslavl, Russia
}

\author{
Article info \\ Received 19.04.2021 \\ Received in revised form 12.05.2021 \\ Accepted 14.05.2021 \\ A. O. Kovalevsky Institute of Biology \\ of the Southern Seas of Russian \\ Academy of Sciences, pr. Leninsky \\ 38/3, Moscow, 119991, Russia. \\ Tel.: +7-978-836-36-56. E-mail. \\ eabocharovainbum@gmail.com \\ Papanin Institute for Biology \\ of Inland Waters Russian Academy \\ of Sciences, Borok village, \\ Yaroslavl region, 152742, Russia. \\ Tel.: +7-978-208-71-85. \\ E-mail:kopytina_n@mail.ru \\ Yaroslavl State Agricultural Academy \\ Tutaevskoe shosse, 58, Yaroslavl, \\ 150042, Russia. \\ Tel.: +7-915-969-98-09. \\ E-mail:elena.slynko.76@mail.ru
}

\begin{abstract}
Bocharova, E. A., Kopytina, N. I., \& Slynko, E. E. (2021). Anti-tumour drugs of marine origin currently at various stages of clinical trials (review). Regulatory Mechanisms in Biosystems, 12(2), 265-280. doi:10.15421/022136

Oncological diseases for a long time have remained one of the most significant health problems of modern society, which causes great losses in its labour and vital potential. Contemporary oncology still faces unsolved issues as insufficient efficacy of treatment of progressing and metastatic cancer, chemoresistance, and side-effects of the traditional therapy which lead to disabilities among or death of a high number of patients. Development of new anti-tumour preparations with a broad range of pharmaceutical properties and low toxicity is becoming increasingly relevant every year. The objective of the study was to provide a review of the recent data about anti-tumour preparations of marine origin currently being at various phases of clinical trials in order to present the biological value of marine organisms - producers of cytotoxic compounds, and the perspectives of their use in modern biomedical technologies. Unlike the synthetic oncological preparations, natural compounds are safer, have broader range of cytotoxic activity, can inhibit the processes of tumour development and metastasis, and at the same time have effects on several etiopathogenic links of carcinogenesis. Currently, practical oncology uses 12 anti-tumour preparations of marine origin (Fludarabine, Cytarabine, Midostaurin, Nelarabine, Eribulin mesylate, Brentuximab vedotin, Trabectedin, Plitidepsin, Enfortumab vedotin, Polatuzumab vedotin, Belantamab mafodotin, Lurbinectedin), 27 substances are at different stages of clinical trials. Contemporary approaches to the treatment of oncological diseases are based on targeted methods such as immune and genetic therapies, antibody-drug conjugates, nanoparticles of biopolymers, and metals. All those methods employ bioactive compounds of marine origin. Numerous literature data from recent years indicate heightened attention to the marine pharmacology and the high potential of marine organisms for the biomedicinal and pharmaceutic industries.
\end{abstract}

Keywords: cancer; marine organisms; preparations; cytotoxic preparations; clinical studies.

\section{Introduction}

Malignant tumors create a significant social problem and have for many years continuned to be the main constituent of non-infectious diseases, causing significant losses of labour and vital potential of society. In 2018 alone, around the world, there $18.1 \mathrm{M}$ new cases of cancer were recorded, $9.6 \mathrm{M}$ cases of death to cancer diseases, $20 \%$ of the population were in the risk group of oncological diseases and $10 \%$ of the patients were highly likely to die (Ferlay et al., 2019). According to the predictions of the experts of the World Health Organization (WHO) and the International Agency for Research on Cancer (IARC), in the period of 2012 to 2030, the global number of cases of cancer found for the first time will increase from $14 \mathrm{M}$ to almost $22 \mathrm{M}$ people, and will grow to 27.5 by 2040 (American Cancer Society (ACS) Global Cancer Facts \& Figures. American Cancer Society; Atlanta, GA, USA: 2018). In some countries of Africa, Asia, Latin America, where many governments do not have enough resources for combating oncological diseases, the growth of morbidity is likely to reach $70 \%$ (Fidler et al., 2018). According to the data of the global statistics, by 2030, in the countries with developed and developping economies, the growth of cancer morbidity will exceed $93 \%$ a year, which is probably related to negative technogenic and anthropogenic factors, and also high level of detection of cancer diseases. In Russia, over $35 \%$ of the patients diagnosed with MT for the first time are of employable age (15-59 years). Now, the number of living people diagnosed with cancer (who have been cured or are currently struggling with the disease) in the RF is approaching $3.5 \mathrm{M}$ people (around $2.3 \%$ of the country's population). If the current situation does not change, every 4th Russian is at the risk of getting cancer, and every 9th may die of it (Petrova et al., 2015; Tjuljandin \& Zhukov, 2018). Tumour diseases hold the leading positions in the mortality level, and at the same time, a colossal amount of resources is spent by the countries around the world annually to treat cancer, diagnose and prevent it at early stages (Savinkova et al., 2018).

Cancer is a whole complex of disorders in the organism, first of all, the neuro-immune-endocrine system. Tumour tissue consists of many associated cells, immunocytes, mediators, hormones, enzymes, molecules of adhesia, mesenchimal and vascular tissues. Furthermore, tumour tissue has a micro-environment and exudes signal molecules that participate in the further process of metastasis. Therefore, the methods of affecting it should be vector-like, targeted as possible, but such that would not damage healthy cells and the tissues.

Efficiency and safety of the therapy of progressive and metastatic cancer diseases, overcoming drug-resistance, combating side-effects of the anti-tumour treatment is a complex and relevant problem in contemporary medicine. Development and study of action of novel anti-tumour compounds with a broad pharmaceutical range and lowest toxicity is important for their further application as monopreparations or combined with traditional chemotherapy. Unlike the synthetic anti-cancer preparations, natural compounds are safer, have a broad range of cytotoxic activity, can inhibit the process of tumour development and metastasis by interacting with several cellular signal pathways and oncogenic factors, making it possible to 
affect several etiopathogenic links of carcenogenesis at the same time and effectively treat various types of tumours (Calcabrini et al., 2017). In this connection, natural marine bioactive compounds are attracting increasing attention.

For a long time, the main approach of the pharmaceutical industry to development of such drugs was based on total screening of biologically active substances obtained from the organisms of various systematic groups of animals. The difficulty of the development of drugs from hydrobionts lies in the problems of provision of a stable raw material basis; the rare occurrence of biological species may complicate obtaining the needed amount of raw material for the development of one or another medical preparation. Despite these difficulties, over the past decade, interest in the natural products of marine origin has greatly increased. Currently, around $60 \%$ of drugs currently used in hematology and oncology are obtained from natural sources. From various marine micro- and macroorganisms, 36,000 new natural compounds have been isolated that are used in therapy of malignant tumours and may be used as promising therapeutic preparations for treatment of various diseases (Dyshlovoy \& Honecker 2015; Barreca et al., 2020). As of the late 2019, in the data base of the National Institutes of Health of the USA, over 5,000 clinical trials have been recorded, and the base PubMed/Medline had over 94,000 scientific articles related to the topics of cancer diseases and preparations of natural origin, including marine, developed or used in practical oncology. High social dependence, distribution of oncopathologies, development of new technologies and approaches to treatment of cancer diseases, demand for multitarget low-toxic anti-tumour preparations have made the development and production of natural bioactive compounds amoung the priority directions in science and the pharmaceutical industry. Therefore, we aimed at making a review of the current data on anti-tumour preparations of marine origin at various stages of clinical trial, demonstrating the biological significance of marine organisms producing cytotoxic compounds and prospects of using them in contemporary biomedical technologies.

The work used the literature data on anti-tumour preparations of marine origin that were approved for the use and/or are underway at phases I-III of clinical trials. The paper also presents bioactive compounds of marine origin, the trials of which demonstrated their cytotoxic activity at phases I-II and are promising for further development of drugs against various types of cancer and other diseases. The review includes the data on biologically active substances isolated from Ascidiacea, sponges, mollusks, Alcyoniina, Bryozoa, marine fungi, cyanobacteria, algae. We analyzed 640 sources of the literature published between 2000 and 2021, and used 268 of them for writing the review.

\section{Marine organisms producing cytotoxic compounds}

Ascidiacea. There are known single and colonial forms that live in coastal and deepwater zones. Ascidians produce a large amount of toxic secondary metabolites that protect them against predators and biofouling organisms. In the period from 1994 to 2014, from ascidians, there were isolated 580 compounds with antibacterial, anti-inflammatory, anti-virulent, anti-diabetes, anti-proliferative, anti-parasitic activities (Palanisamy et al., 2017). Currently, the most efficient producers of biologically active compounds are considered the representatives of the Didemnidae, Polyclinidae and Polycitoridae families, and out of 69 representatives of Didemnum genus, there were isolated 212 biologically active compounds (Diaa et al., 2020). The high pharmacological potential of nonribosomal peptides, proline-rich cyclic peptides and many compounds of various chemical classes produced by ascidians, and clinical application of them for the treatment of broad range of diseases, including in oncology, are well known (Diaa et al., 2020). In experiments, toxic metabolites of ascidians exerted effects on DNA transcription, protein translation, processes of neurohumoral regulation, cytoskeleton (Agrawal et al., 2016; Fang et al., 2016; Negi et al., 2017; Arumugam, 2018). Practical medicine uses 4 drugs derived from ascidians to treat patients suffering oncological diseases: Trabectidin, Aplidin ${ }^{\circledR}$, Midostaurin, Lurbinectedin.

Sponges are aquatic, chiefly marine multicellular animals that live attached to surfaces, without any true organs. Sponges have no intrinsic immune system or structures of mechanical protection such as shell or the spinal cord. Therefore, their only way to protect themselves is to produce active metabolites that act as a means of protection against and adaptation to the environment, they have effective systems of biotransformation and detoxication that counteract the influence of DNA-damaging carcinogens (Aiub et al., 2006.). There are known 53,000 active compounds isolated from sponges and their associating organisms (Bibi et al., 2017). In the period 2001-2010, up to $30 \%$ of identified biologically active substances were isolated from them: avarols, arenastatins, halichondrin, milnamid, spongistatin, dactylolides, dictiostatin, discodermolide, hemiasterlin, laulimalide, peloruside A and zampanolide (Mehbub et al., 2014; Gnanambal \& Lakshmipathy, 2016; Santos et al., 2020). Currently, 774 new compounds isolated from marine sponges and associating microorganisms have been studied and described, and $42 \%$ of them demonstrated high biological activity (Cheng et al., 2020). Preparations developed based on those compounds are efficient in treatment of metastatic cancer of the mammary gland, lymphomas. Bioactive compounds also exerted in vitro anti-tumour activity against other types of cancer cells (Ercolano, 2019) resulting from numerous cellular and molecular mechanisms that include protection of DNA, modulation of the cellular cycle, processes of apoptosis and antiinflammatory activity (Calcabrini et al., 2017). Pro-apoptotic mechanisms of many compounds are not completely clear, but, nonetheless, they are known to induce several apoptotic pathways at the same time. Practical oncology uses 4 anti-tumour preparations made of marine sponges: Fludarabine, Cytarabine, Nelarabine, Eribulin mesylate.

Mollusks are broadly distributed in marine, freshwater bodies and soil. Chemical examinations of marine mollusks revealed a broad spectrum of bioactive substances produced by these organisms in order to adapt to and survive in various environments - peptides, sterines, polyketides, carotenoids, terpenes, polypropionates, macrolides, polyunsaturated fatty acids, alkaloids, nitrogen compounds, etc. According to the data of 2014, 1,145 biologically active compounds were isolated from marine mollusks, and in the period between 2014 and 2018, another 145 substances were added to this list (Summer et al., 2020). Because of the variety of chemical structures and broad range of biological activity, these substances have high cytotoxic potential (Ciavatta et al., 2017; Ahmad et al., 2018; Esmaeelian et al., 2018; Avila \& Angulo-Preckler, 2020). Many anti-tumour compounds reached late pre-clinical and clinical developments (Newman \& Cragg, 2014). The high efficacy of anti-tumour bioactive compounds isolated from mollusks is due to their high selectivity, ability to overcome chemoresistance, alter biological characteristics of cancer cells, mechanisms of activation of caspases that take part in interal and external cellular apoptotic pathways, inhibition of antiogenesis, imbalance of tubulin and microtubes (Chakraborty et al., 2020). Oncological practice uses 4 preparations of mollusks: Belantamab mafodotin, Polatuzumab vedotin, Enfortumab vedotin, Brentuximab vedotin.

Bryozoa are aquatic colonial animals, a poorly studied group (around 6,000 species are known). Many species are broadly distributed in the tropics and subtropics, the colonies have a large biomass. The amount of known biologically active substances from Bryozoa is comparatively low. This is due to the fact that the biologically active compounds have until now been being extracted by the traditional techniques, using organic solvents, with great complexity of process. The studies at different stages of clinical and pre-clinical trials demonstrated the effectiveness of active metabolites of Bryozoa, which have cytotoxic, antiparasitic, antiviral, cognitive-restoring, antidepressive, anti-spasm activities. (Ciavatta et al., 2020). About 260 medical preparations were obtained from 23 species of Bryozoa (Carroll et al., 2020). Currently, the problems of extraction and synthesis of biologically active cytotoxic compounds from Bryozoa are being broadly discussed, which gives us hope for more thorough study and use of their beneficial metabolites in practical medicine (Gomes et al., 2016).

Coral reefs are recognized as the most dynamic and variable biosystem on the planet, which contains many studied and unstudied organisms (Moeller et al., 2019). Corals are hosts of species-specific microbial communities, including bacteria and fungi that produce bioactive substances, mainly of cytotoxic activity. From coral-associated organisms, 300 biologically active substances with cytotoxic, antiparasitic, antibacterial, antiinflammatory activities have been isolated (Hou et al., 2019). These compounds are studied as potential anti-tumour and antiviral preparations, including against SARS-CoV-2 and HIV (El-Hossary et al., 2020; Zahran et al., 2020). Many bioactive products (terpenoids, sesquiterpenes, diterpe- 
nes, steroids and many other) are produced by soft corals and are promising objects for the development of various pharmaceutical preparations (Sang et al., 2019).

Marine fungi are a group of heterotrophic organisms that have traits of plants and animals. Over 1,100 species of microscopic fungi isolated are known from the marine environment, whose ancestors may have evolved in freshwater water bodies and terrestrial living places (Jones et al., 2015). The same species of fungi isolated from terrestrial and marine ecosystems synthesize various biologically active compounds (Pivkin et al., 2006.; Deshmukh et al., 2018). Some substances isolated from fungi of marine origin can exert notable anti-tumour activity through various mechanisms such as apoptosis and induction of the suspencion of cellular cycle (Evidente et al., 2014). The major fungal producers of the natural products are recognized to be marine fungi of genera Aspergillus - 28\%, and Penicillum - 11\%. (Liu et al., 2020; Fadia et al., 2021; Youssef et al., 2021). Secondary metabolites of marine fungi include peptides, alkaloids, terpenoids, steroids, lactones, polyketides (Alves et al., 2019; Ogaki et al., 2020; El-Kashef et al., 2021). A total of 53\% of the examined substances mostly displayed cytotoxic, antimicrobial and antiviral activities, and to a lower degree demonstrated anti-diabetes and hypolipidemic actions. Active metabolites exhibited high bioavailability during peroral introduction, and at the same time are less toxic for humans compared with their synthesized analogues (Youssef et al., 2019). The advantage of fungi is that they may be cultivated with high rate of reproduction.

Cyanobacteria or blue-green algae are the oldest complexely organized procariotic microorganisms that live in water. Cyanobacteria are a rich source of known and new biologically active compounds with strong medical potential (Shah et al., 2017). These organisms are able to produce efficient toxins and other secondary metabolites, including polypeptides, polyketides, alkaloids, lipids, polysaccharides, swinholides, terpenes with notable bioactive properties. Thus, dolastatin 10, obtained from some species of cyanobacteria and the marine mollusk Dolabella auricularia, gave a start to the production of its synthetic analogues - MMAE and MMAF, used in modern antibody-drug conjugates. They are aimed at cancer cells, causing their apoptotic death or affecting the processes of signal cellular transmission. Numerous studies demonstrated antibacterial, antifungal, anti-tuberculosis, immune-depressing and anti-inflammatory properties of these bioactive compounds (Vijayakumar \& Menakha, 2015; Rojas et al., 2020; Demay et al., 2021). Several species of cyanobacteria, cultivated on commercial platforms to obtain bioactive substances, are used in contemporary nanotechnological systems of selective transport of drugs to the tumours (Qamar et al., 2021).

Microalgae are a group of phototrophic organisms, which includes a large number of species of single-cell algae with broad range of distribution (marine and freshwater bodies, soils). Marine algae are recognized as a rich source of various biologically active substances (Cheung et al., 2015; Martínez Andrade et al., 2018). Biopolymers of marine algae are known for their broad range of pharmacological effects: cytostatic, antiproliferative, antimetastatic, proapoptotic, antiangiogenic, and immunemodulating, having at the same time low toxicity. Bioactive substances of microalgae can inhibit all phases of carcenogenesis, making them a promising source of original anti-tumour medical and chemoprevention preparations (Galasso et al., 2019). Cancer preventive effects of the compounds of marine algae are associated with immunogenic properties (Sansone et al., 2021). Therefore, fucoidan, sulfated polysaccharides (dopolan and supolan), carrageenans and pectins stimulate the work of the complement system, processes of phagocytosis, synthesis of immunoglobulins, mechanisms of cellular defence, particularly production and differentiation of T-lymphocytes and natural killers, increase the level of immunoglobulin A. The advantages of microalgae used for the development of medical preparations are their metabolic plasticity and possibility of their cultivation in photobioreactors, obtaining a larger amount of biomass. Currently, commercial production of microalgae is around 5,000 tonnes of dry mass a year (Abidizadegan et al., 2020). They are recognized as promising objects for scientific developments in the pharmaceutical and biomedical industries, the food industry, in production of biomaterials, biofuel (Balasubramaniam et al., 2021; Remize et al., 2021).

The most important role in life of the host organism is played by fungi, bacteria, viruses and microalgae, by providing food, protection, and re- production. It was determined that many active substances are produced not by marine organisms, but endosymbiotic microorganisms associated with them (Gerwick \& Moore, 2012; McCarthy et al., 2019). Compounds exuded by symbionts, such as sterols, alkaloids, peroxides, terpenes, fatty acids, derivatives of aminoacids, cyclic peptides, depsipeptides, polyketides, xanthones, toxins, bryostatins are highly biologically active. For example, Actinobacteria, especially those of the Streptomyces genus, produce around 100 natural products, which provide different types of biological defence to their host organisms. The most productive symbionts are considered Actinobacteria, Proteobacteria, Bacteroidetes, Firmicutes, Cyanobacteria (Zhang et al., 2017; Gavriilidou et al., 2021). Marine associative algae, other than having broadly known medical-preventive and biopharmaceutical values, produce substances that inhibit the growth of pathogenic bacteria and viruses that affect the vital processes of host organisms, including industrially valuable mollusks and fish (Riccio et al., 2020). Marine fungi live in symbiosis with various microbial communities, their biological properties are well known, but the potential of associating organisms remains studied incompletely. Recent surveys of 450 chemical compounds of nudibranchs and symbionts, which are some of the most chemically diverse groups, have revealled their impressive bioactive potential (Avila \& Angulo-Preckler, 2020).

To identify the most bioproductive communities and their pharmacological properties, genetic surveys are being carried out (Knobloch et al., 2020). New technologies of growing symbiotic organisms of sponges are being developed for the purposes of cultivation and further obtaining of isolates of the dominant and most productive microbial associates in the pharmaceutical industry (Knobloch et al., 2019). For instance, 1,200 new bioactive compounds were reported to be identified from Ascidiacea, but recent studies have revealed that biologically efficient products are synthesized by Ascidiacea to a lower degree than symbiont microorganisms (Watters, 2018; Dou \& Dong, 2019). Corals provide symbiotic co-existance of mollusks, crustaceans, sponges, fishes, worms, viruses, bacteria, algae, fungi; however, symbiotic communities of associating organisms of coral reefs are so far studied insufficiently (Blackall et al., 2015; Kellogg, 2019). Viral, bacterial, fungal associanting organisms provide physiological stability of corals and resistance of the system to various stressor effects by producing toxic bioactive compounds, antibiotics, which destroy pathogens through lysis, affecting the composition of holobiont and production of secondary metabolites by symbionts (Roach et al., 2020). Symbiotic communities produce many powerful compounds (carbohydrates, exopolysaccharides, lipids, peptides, alkaloids, polyphenols, steroids, polyketides, terpenoids and zoanthoxanthin), which have cytotoxic, anti-tumour, neuroprotective, antiparasitic, antibacterial effects, and constitute the richest source of new natural compounds with varied chemical structure, high biochemical, pharmacotherapeutic and industrial potentials (Yang et al., 2018; Peter et al., 2019; Madalena et al., 2021).

\section{Antibody-drug conjugates of marine origin with beneficial loads}

Against various types of malignant tumours, standard methods of chemotherapy are the only approaches to treatment. Classic medical preparations affect the carcinogenesis through various mechanisms: by neutralizing antigene proteins, blocking their endothelial receptors, inhibiting synthesis of proteins by cancer cells, directly inducing apoptosis of endothelial cells, destroying the tubulin and vascular network of tumours (Pérez-Pérez et al., 2016). However, such preparations damage healthy cells as well, causing the patients to suffer heavy side-effects, and the toxic impact is first of all suffered by the cells of the mucous membrane of the gastrointestinal tract, reproductive and immune systems, and hair. Modem methods of targeted anti-tumour therapy can be aimed at cancer cells. They include the comparatively recently developed system of antibodydrug conjugates, which allow a target impact on malignant tumours, thus making it less harmful for healthy cells.

Antibodies are large molecules of the immune system, which are able to develop specific relationships with the cellular surface of an "alien" object, and eliminate it through complex processes. This natural mechanism, intrinsic in the organism, is a principle of the work of antibody-drug conjugates (or "immune-conjugants"). In clinical practice, monoclonal antibodies demonstrated a large potential and efficiency as new classes of prepa- 
rations. An antibody-drug conjugate (ADC) is a three-component molecule that consists of antibody (unit of selective targeting), medical cytotoxical preparations (beneficial loads) and chemical/peptide linker that combines them. The antibody provides conjugant directly to affected cells, interacts with specific tumour markers, protein-antibody expressed only by tumour cells. Then, ADC internalizes, and then, when introduced to the cell, there occurs breakdown of the linker, release of medical agent that suspends mitosis and apoptosis of cancer cells. Therefore, the toxin, strongly bound with the antibody, enters the tumour cell, practically not damaging the healthy tissue.

As beneficial loads, half of the most often used ADCs contain auristatins (monomethyl auristatin A and monomethyl auristatin F), powerful anti-tubulin agents, synthetic analogues of marine dolostatin 10 , which are cytotoxic ultrapotent inhibitors of microtubules (Johansson et al., 2017). Monomethyl auristatin E (MMAE, vedotin) is an effective anti-mitotic preparation that inhibits splitting of cells through blocking polymerization of tubulin. MMAE exceeds the cytotoxicity of doxorubicin (adriamycin/ rubex) by 100-1000 times. Therefore, it is not used as an individual medical preparation, and is applied as beneficial medical load in the content of ADC. Monomethyl auristatin F (MMAF) is a powerful inhibitor of tubuline polymerase. It is different from MMAE because the residual of norephedrine is replaced by phenylalanine and because of the lower penetrability to the cells. By overcoming internal resistance of the tumour, auristatins increase sensitivity of cells to ionizing emission, which improves the quality of X-ray surveillance of the tumour process and increases the therapeutic efficacy of impenetrable radiation-sensitizing agents during $X$ ray therapy (Bourillon et al. 2019; Hingorani et al., 2020). Studies of ADC with dolastatins, other than the generally known direct cytotoxic effect, demonstrate such effects as induction of homing of dendritic cells and activation of the system of cellular anti-tumour immunity (Müller et al., 2014). The unique quality of $\mathrm{ADC}$ is the antitumour impact combining immune- and chemotherapy in one preparation (Choi et al., 2015). Their efficiency depends on every component separately, as well as their cohe- rent interaction, which includes many internal and external cellular processes. Nine ADCs have been approved, over 16 have passed the clinical trials, over 60 are being studied at various phases, and numerous research projects on studying and improving the properties and overcoming mechanisms of resistance to them are underway (Greene et al., 2020; Zhao et al., 2020).

Having adapted to variable and not always favourable conditions of existence in the marine environment, marine organisms synthesize many toxic compounds, which are different from biologically active terrestrial analogues. Unlike their synthetic derivatives, they have a more complex chemical structure, thus demonstrating more interesting, and often outstanding chemical properties (Jimenez et al., 2020). Many of the abovementioned marine organisms produce unique biochemical substances, making them quite promising for the development of new pharmaceutical preparations with multi-target therapeutic effects. Thus, marine organisms produce natural biologically active products, broadly used in many spheres of science, practical medicine and industry.

Currently, 12 preparations are in use obtained from marine organisms, which were approved by the FDA (Food and Drug Administration of the USA) and EMEA (European Medicines Agency - agency dealing with evaluation of medical preparations for their correspondence to the requirements written in the European Pharmacopoeia) and registered as medicinal preparations for therapy of oncological patients (Fludarabine, Cytarabine, Midostaurin, Nelarabine, Eribulin mesylate, Brentuximab vedotin, Trabectedin, Plitidepsin, Enfortumab vedotin, Polatuzumab vedotin, Belantamab mafodotin, Lurbinectedin), a total of 27 compounds are being tested at various phases of clinical trials (Table 1). We used the recent data about the clinical trials from the updating official sources of the EU and USA (www.clinicaltrials.gov; www.clinicaltrialsregister.eu).

Cytotxic substances, trials of which were completed or stopped at the intermediate phases, demonstrated their anti-tumour effects. Studies of their therapeutic properties are likely to be continued against various types of tumours and other diseases (Table 2).

Table 1

Anti-tumour preparations from marine organisms currently undergoing various phases of clinical trials

\begin{tabular}{|c|c|c|c|c|}
\hline $\begin{array}{l}\text { Name of drug/ } \\
\text { Company }\end{array}$ & $\begin{array}{l}\text { Marine organism/ } \\
\text { chemical class }\end{array}$ & Purpose, target & Mechanism of action & Reference \\
\hline \multicolumn{5}{|c|}{ Preparations approved for use and at various phases of clinical trials against different types of cancer } \\
\hline $\begin{array}{l}\text { Fludarabine (Vidarabine, Fludara, } \\
\text { F-ara-AMP, Ad/PNP-F-araAMP/ } \\
\text { PNP) / Therapeutics, Inc. }\end{array}$ & Fungi/Nucleoside & Leukoses, lymphomas & $\begin{array}{l}\text { Cytostatic of the group of purine antagonists, inhibitor } \\
\text { of key DNA enzymes, apoptosis inductor }\end{array}$ & $\begin{array}{l}\text { Lowe et al., 2018; } \\
\text { Barreca et al., 2020; } \\
\text { Cooper et al., 2020. }\end{array}$ \\
\hline $\begin{array}{l}\text { Cytarabine (ARA-C, Cytozar-U }{ }^{\mathbb{R}} \text {, } \\
\text { DepoCyt }^{\mathbb{R}} \text { )/ Pfizer }\end{array}$ & Fungi/Nucleoside & Leukoses & $\begin{array}{l}\text { Inhibitor of DNA-polymerase, damages DNA } \\
\text { in S-phase of cellular cycle }\end{array}$ & $\begin{array}{l}\text { Salehi et al., 2019; } \\
\text { Frankel et al., 2020. }\end{array}$ \\
\hline $\begin{array}{l}\text { Nelarabine(Arranon)/ } \\
\text { Washington University School of } \\
\text { Medicine }\end{array}$ & Fungi/Nucleoside & Leukoses, lymphomas & $\begin{array}{l}\text { Purine antimetabolite, accumulates mostly in T-cells, } \\
\text { mechanism of action is similar to cytarabine }\end{array}$ & $\begin{array}{l}\text { Kadia et al., 2017; } \\
\text { Barreca et al., 2020; } \\
\text { Dunsmore et al., 2020. }\end{array}$ \\
\hline $\begin{array}{l}\text { Midostaurin (PKC 412)/ Novartis } \\
\text { Pharmaceuticals, Stichting } \\
\text { Hemato-Oncologie voor } \\
\text { Volwassenen Nederland }\end{array}$ & $\begin{array}{l}\text { Ascidiacea, Actinobacteria } \\
\text { / Indolocarbazole }\end{array}$ & $\begin{array}{l}\text { Acute myelogenous } \\
\text { leukemia, hematologic } \\
\text { cancer }\end{array}$ & $\begin{array}{l}\text { Multi-target inhibitor of protein kinase } 3 \text { (FLT 3), } \\
\text { active against oncogenic CD } 135\end{array}$ & $\begin{array}{l}\text { Barreca et al., 2020; } \\
\text { Orgueira et al., 2020; } \\
\text { Morell et al., 2021. }\end{array}$ \\
\hline $\begin{array}{l}\text { Eribulin mesylate (Halichondrin } \\
\text { B, E7389, Halaven }{ }^{\mathbb{B}} \text { )/ Eisai Inc. }\end{array}$ & Fungi/Macrolide & $\begin{array}{l}\text { Refractory solid tumours, } \\
\text { Ewing's sarcoma, metasta- } \\
\text { tic mammary gland cancer }\end{array}$ & $\begin{array}{l}\text { Non-taxane inhibitor of dynamics of microtubules } \\
\text { with high anti-mitotic activity }\end{array}$ & $\begin{array}{l}\text { Barroso-Sousa et al., 2020; } \\
\text { Tolaney et al., 2020; } \\
\text { Yuan et al., 2021. }\end{array}$ \\
\hline $\begin{array}{l}\text { Brentuximab vedotin (SGN 35, } \\
\text { Adcetric }^{\circledR} \text { )/ Seattle Genetics }\end{array}$ & $\begin{array}{l}\text { Mollusks, cyanobacteria / } \\
\text { ADC with MMAE }\end{array}$ & $\begin{array}{l}\text { T-cellular lymphomas, } \\
\text { Hodgkin lymphoma }\end{array}$ & $\begin{array}{l}\text { First target ADC-preparation. } \\
\text { Targeted at CD30-positive tumour cells, microtubules }\end{array}$ & $\begin{array}{l}\text { Chen et al., 2020; } \\
\text { Straus et al., } 2020 \text {. }\end{array}$ \\
\hline $\begin{array}{l}\text { Trabectedin }\left(\text { ET-743, Yondelis }{ }^{(B)}\right) / \\
\text { Pharmamar }\end{array}$ & Ascidiacea/Alkaloid & $\begin{array}{l}\text { Progressive liposarcoma, } \\
\text { metastatic sarcomas of the } \\
\text { soft tissues }\end{array}$ & $\begin{array}{l}\text { Pleiotropic mechanism of action, has effect on the key } \\
\text { cellular processes and micro-environment of tumour }\end{array}$ & $\begin{array}{l}\text { Miwa et al., 2019; Kobaya- } \\
\text { shi et al., 2020; Martínez- } \\
\text { Trufero et al., 2021. }\end{array}$ \\
\hline $\begin{array}{l}\text { Plitidepsin (dehydrodidemnin B, } \\
\text { Aplidin }^{\circledR} \text { )/ PharmaMar }\end{array}$ & $\begin{array}{l}\text { Ascidiacea/ } \\
\text { Cyclodepsypeptide }\end{array}$ & $\begin{array}{l}\text { Medulloblastoma, leukoses } \\
\text { and liposarcomas; MCF-7 }\end{array}$ & $\begin{array}{l}\text { Specifically binds with eEF1A2, activates RAC1 and } \\
\text { phosphorylation of JNK1, induces apoptosis depend- } \\
\text { ing on the type of cell and dose. Inhibitor of microtu- } \\
\text { bule assembly. Regulates expression of growth factor } \\
\text { gene of the blood vessels of the endothelium and } \\
\text { blocks its secretion }\end{array}$ & $\begin{array}{l}\text { Popov, 2006; Xu et al., } \\
\text { 2012; Newman \& Cragg, } \\
\text { 2014; Alonso-Álvarez et } \\
\text { al., 2017; Xing et al., 2017; } \\
\text { Martínez Andrade et al., } \\
\text { 2018; Pereira et al., 2019; } \\
\text { Losada et al., 2020. }\end{array}$ \\
\hline $\begin{array}{l}\text { Enfortumab vedotin (AGS- } \\
\text { 22MSE)/ Agensys, Seattle } \\
\text { Genetics, Astellas Pharma }\end{array}$ & $\begin{array}{l}\text { Mollusks, cyanobacteria/ } \\
\text { ADC with MMAE }\end{array}$ & $\begin{array}{l}\text { Multiple solid tumours, } \\
\text { metastatic cancers of the } \\
\text { urinary system and the } \\
\text { mammary glands }\end{array}$ & $\begin{array}{l}\text { The first and the only agent aimed at nectin- } 4 \text {, } \\
\text { molecule of cellular adhesion, which expresses on } \\
\text { many solid tumours }\end{array}$ & $\begin{array}{l}\text { Newman, 2019; } \\
\text { Pereira, 2019; } \\
\text { Hanna, 2020. }\end{array}$ \\
\hline
\end{tabular}




\begin{tabular}{|c|c|c|c|c|}
\hline $\begin{array}{l}\text { Name of drug/ } \\
\text { Company }\end{array}$ & $\begin{array}{l}\text { Marine organism/ } \\
\text { chemical class }\end{array}$ & Purpose, target & Mechanism of action & Reference \\
\hline $\begin{array}{l}\text { Polatuzumab vedotin (Polivy TM, } \\
\text { DCDS4501A, RG7596)/ } \\
\text { Genentech, Roche }\end{array}$ & $\begin{array}{l}\text { Mollusks, cyanobacteria/ } \\
\text { ADC with MMAE }\end{array}$ & $\begin{array}{l}\text { Recurrent or refractory } \\
\text { diffusive large B-cell } \\
\text { lymphoma }\end{array}$ & $\begin{array}{l}\text { Selectively binds with CD79b on the surface } \\
\text { of B-cells, inhibits polymerization of tubulin, } \\
\text { suspends phase G2 / M and apoptosis of tumour cells }\end{array}$ & $\begin{array}{l}\text { Wang, 2017; Nejadmog- } \\
\text { haddam et al., 2019; Pereira } \\
\text { et al., 2019; Amaya et al., } \\
\text { 2020; Choi \& Diefenbach, } \\
\text { 2020. }\end{array}$ \\
\hline $\begin{array}{l}\text { Belantamab mafodotin } \\
\left.\text { (GSK2857916, Blenrep }^{\mathrm{TM}}\right) / \\
\text { GlaxoSmithKline }\end{array}$ & $\begin{array}{l}\text { Molusks, cyanobacteria/ } \\
\text { ADC with MMAE }\end{array}$ & $\begin{array}{l}\text { Recurrent and refractory } \\
\text { multiple myeloma }\end{array}$ & $\begin{array}{l}\text { Afucosylated antibody IgG1 aimed at antigen } \\
\text { maturation of B-cells (BCMA) }\end{array}$ & $\begin{array}{l}\text { Pereira et al., 2019; } \\
\text { Tzogani et al., 2020; } \\
\text { Musaimi et al., 2021. }\end{array}$ \\
\hline $\begin{array}{l}\text { Lurbinectedin (LY-01017, } \\
\left.\text { PM-01183, Zepsyre, Zepzelca }{ }^{\mathrm{TM}}\right) / \\
\text { PharmaMar }\end{array}$ & Ascidiacea/Alkaloid & $\begin{array}{l}\text { Sarcomas of the soft tis- } \\
\text { sues, chronic lymphocytic } \\
\text { leukemia. Orphan drug for } \\
\text { ovarian cancer, small -cell } \\
\text { lung cancer }\end{array}$ & $\begin{array}{l}\text { Inhibits the processes of oncogenic transcription, } \\
\text { covalently binding with DNA in the tumor tissues, } \\
\text { stimulates anti-tumor immunity, directly affects the } \\
\text { micro-environment of tumours }\end{array}$ & $\begin{array}{l}\text { Popov, 2006; Takahashi } \\
\text { et al., 2016; Belgiovine } \\
\text { et al., 2017; Calvo et al., } \\
\text { 2017; Martínez Andrade, } \\
\text { 2018; Kauffmann-Guerre- } \\
\text { ro, 2020; Markham, } 2020 .\end{array}$ \\
\hline \multicolumn{5}{|c|}{ Phase III } \\
\hline $\begin{array}{l}\text { Plinabulin (NPI-2358)/Nereus } \\
\text { Pharmaceutical }\end{array}$ & $\begin{array}{l}\text { Marine fungi/ } \\
\text { Diketopiperazine }\end{array}$ & $\begin{array}{l}\text { Multiple myeloma, } \\
\text { adenocarcinoma of the } \\
\text { large intestine, prostate, the } \\
\text { mammary glands }\end{array}$ & $\begin{array}{l}\text { Blocks polymerization of tubulin through unique } \\
\text { mechanism, leading to multi-factor effects, intensified } \\
\text { immune-oncologic response and disruption of blood } \\
\text { support of tumour }\end{array}$ & $\begin{array}{l}\text { Singh et al., 2011; Martínez } \\
\text { Andrade et al., 2018; } \\
\text { Pereira, 2019; Jimenez, } \\
\text { 2020; Tonra et al., 2020. }\end{array}$ \\
\hline $\begin{array}{l}\text { Marizomib (SalinosporamideA. } \\
\text { NPI0052)/Nereus } \\
\text { Pharmaceutical, Celgene, Triphase }\end{array}$ & $\begin{array}{l}\text { Actinomycetales / } \\
\gamma \text {-lactam- } \beta \text {-lactone }\end{array}$ & $\begin{array}{l}\text { Glioblastoma, carcinoma } \\
\text { of large intestine, CNS } \\
\text { tumour, melanoma, non- } \\
\text { small-cell lung cancer, } \\
\text { mammary glands cancer }\end{array}$ & $\begin{array}{l}\text { The only inhibitor of activity of proteasomes, which } \\
\text { can overcome blood-brain barrier. Covalently } \\
\text { modifies the residues of teonin, active site of } 20 \mathrm{~S}- \\
\text { proteasome }\end{array}$ & $\begin{array}{l}\text { Martins et al., 2014; } \\
\text { Jensen et al., 2015; Pérez- } \\
\text { Pérez et al., 2016; Pereira } \\
\text { et al., 2019; Roth et al., } \\
\text { 2020; Sherman \& Li, } 2020 .\end{array}$ \\
\hline \multicolumn{5}{|c|}{ Phases II/III } \\
\hline $\begin{array}{l}\text { Depatuxizumab mafodotin } \\
\text { (depatux-m, ABT-414, ABT 806)/ } \\
\text { Abbvie }\end{array}$ & $\begin{array}{l}\text { Mollusks, cyanobacteria / } \\
\text { / ADC with MMAF }\end{array}$ & $\begin{array}{l}\text { Squamous cell cancer } \\
\text { of the lungs, glioblastoma } \\
\text { multiforme }\end{array}$ & $\begin{array}{l}\text { Binds EGFR epitope on the surface of cell, interna- } \\
\text { lizes amd releases cys-mcMMAF (cys-mafodotin), } \\
\text { binds with the network of microtubules, suspending } \\
\text { the proliferation of tumour cells }\end{array}$ & $\begin{array}{l}\text { Pereira et al., 2019; } \\
\text { Biteghe et al., 2020; } \\
\text { Van Den Bent et al., } 2020 .\end{array}$ \\
\hline \multicolumn{5}{|c|}{ Phase II } \\
\hline $\begin{array}{l}\text { Oligo-fucoidan / Hi-Q Marine } \\
\text { Biotech International, Ltd. }\end{array}$ & $\begin{array}{l}\text { Brown marine algae/ } \\
\text { Polysaccharide }\end{array}$ & $\begin{array}{l}\text { Metastatic colorectal } \\
\text { cancer, hepatocellular } \\
\text { carcinoma, mammary } \\
\text { glands cancer, lung cancer }\end{array}$ & $\begin{array}{l}\text { Has effect on receptor of transformation growth factor } \\
\text { (TGF- } \beta \text { ), inhibits epithelial-mesenchymal transition } \\
\text { in tumour cells }\end{array}$ & $\begin{array}{l}\text { Tsai et al., 2017; } \\
\text { Lin et al., 2020; } \\
\text { Miyata et al., } 2020 .\end{array}$ \\
\hline $\begin{array}{l}\text { Tisotumab vedotin (Hu Max }{ }^{\mathrm{R}}-\mathrm{TF}- \\
\text { ADC, TF-011-MMAE)/ } \\
\text { Genmab, Seattle Genetics }\end{array}$ & $\begin{array}{l}\text { - Mollusks / ADC with } \\
\text { MMAE }\end{array}$ & $\begin{array}{l}\text { Metastatic cervical cancer, } \\
\text { endometrial cancer, bladder } \\
\text { cancer, prostate cancer, eso- } \\
\text { phagus cancer, squamous } \\
\text { cell carcinoma of the head } \\
\text { and neck }\end{array}$ & $\begin{array}{l}\text { The first ADC in its class, which contains human } \\
\text { tissue factor (TF)-specific monoclonal antibody. } \\
\text { Inhibitor of microtubules, immune-toxin }\end{array}$ & $\begin{array}{l}\text { De Bono et al., 2019; } \\
\text { Newman, 2019; } \\
\text { Pereira et al., 2019; } \\
\text { Hong et al., 2020. }\end{array}$ \\
\hline $\begin{array}{l}\text { AGS-16C3F (AGS-16M8F)/ } \\
\text { AgenYS Inc, Astellas Farma Inc. }\end{array}$ & $\begin{array}{l}\text { Mollusks, cyanobacteria/ } \\
\text { ADC with MMAE }\end{array}$ & $\begin{array}{l}\text { Carcinoma of the liver, } \\
\text { metastatic hepatocellular } \\
\text { cancer }\end{array}$ & $\begin{array}{l}\text { Inhibitor of tubulin polymerase, tubulin, mytosis; } \\
\text { apoptosis stimulator. Targets phosphodiester } 3 \text {, } \\
\text { ectonucleotide pyrophosphatase (ENPP3) }\end{array}$ & $\begin{array}{l}\text { Newman \& Cragg, 2017; } \\
\text { Gogineni \& Hamann, 2018; } \\
\text { Pereira et al., 2019; Koll- } \\
\text { mannsberger et al., } 2021 .\end{array}$ \\
\hline $\begin{array}{l}\text { Telisotuzumab vedotin } \\
(\mathrm{ABBV}-399) / \mathrm{ABBVie}\end{array}$ & $\begin{array}{l}\text { Mollusks, cyanobacteria / } \\
\text { ADC with MMAE }\end{array}$ & $\begin{array}{l}\text { Recurring and stage IV } \\
\text { of lung cancer, advanced } \\
\text { cases of cancer }\end{array}$ & $\begin{array}{l}\text { The first ADC in its class that contains humanitized } \\
\text { monoclonal antibody ADT } 700 \text {. Aimed at c-Met } \\
\text { expressing tumour cells }\end{array}$ & $\begin{array}{l}\text { Strickler et al., 2018; } \\
\text { Newman, 2019; } \\
\text { Pereira et al., 2019. }\end{array}$ \\
\hline $\begin{array}{l}\text { Dolastatin } 10 \text { and } 15 / \\
\text { Seatle Genetics }\end{array}$ & $\begin{array}{l}\text { Mollusks, cyanobacteria/ } \\
\text { Linear pentapeptide }\end{array}$ & $\begin{array}{l}\text { Mammary cancer, cancers } \\
\text { of the large intestine, the } \\
\text { liver, solid tumours and } \\
\text { some leukoses }\end{array}$ & $\begin{array}{l}\text { Obstructs formation of tubulin, induces apoptosis and } \\
\text { phosphorylation of Bcl- } 2 \text { in some types of malignant } \\
\text { cells. Suspends cellular cycle in phase G2-M }\end{array}$ & $\begin{array}{l}\text { Perez et al., 2005; } \\
\text { Martínez Andrade et al., } \\
\text { 2018; Yang et al., 2019; } \\
\text { Ratnayake et al., 2020. } \\
\end{array}$ \\
\hline \multicolumn{5}{|c|}{ Phases I/II } \\
\hline $\begin{array}{l}\text { KRN } 7000 \text { (Agelasphin } \\
\text { derivate)/ Shanghai Public } \\
\text { Health Clinical Center }\end{array}$ & $\begin{array}{l}\text { Fungi/ } \\
\alpha \text {-Galactosylceramide }\end{array}$ & $\begin{array}{l}\text { Cancers of the lungs, } \\
\text { stomach, kidneys, liver }\end{array}$ & $\begin{array}{l}\text { Inductor of stable proliferation and production of } \\
\text { interleukins of iNKT cells }\end{array}$ & $\begin{array}{l}\text { Fuji et al., 2000; Anderson } \\
\text { et al., 2013; Hartrampf et al., } \\
\text { 2020; Washah et al., 2020. }\end{array}$ \\
\hline $\begin{array}{l}\text { Cirmtuzumab vedotin } \\
\text { (UC961ADC3)/ UC San Diego }\end{array}$ & Various / ADC with MMAE & $\begin{array}{l}\text { Solid tumours, B-cellular } \\
\text { lymphocytic leukemia, } \\
\text { mammary cancer }\end{array}$ & $\begin{array}{l}\text { Targets epitope of ROR1. Competative inhibitor of } \\
\text { binding ligand WNT, interrupts signal cascade } \\
\text { associated with effects of ROR1 }\end{array}$ & $\begin{array}{l}\text { Choi et al., 2015; } \\
\text { Choi et al., 2018; } \\
\text { Menck et al., 2021. }\end{array}$ \\
\hline $\begin{array}{l}\text { XMT 1536(Upifitamab } \\
\text { rilsodotin)/ Mersana } \\
\text { Therapeutics, IQVIABiotech }\end{array}$ & $\begin{array}{l}\text { Mollusks, cyanobacteria/ } \\
\text { ADC Dolaflexin with } \\
\text { MMAF }\end{array}$ & $\begin{array}{l}\text { Platinum-resistant ovarian } \\
\text { cancer, metastatic non-small- } \\
\text { cell lung cancer, tumours that } \\
\text { express NaPi2b }\end{array}$ & $\begin{array}{l}\text { Novel technology of ADC, contains higher } \\
\text { concentration of medical antibody and novel auristan } \\
\text { F- hydroxypropylamine (AF-HPA). Targeted at Her2, } \\
\text { NaPi2b }\end{array}$ & $\begin{array}{l}\text { Manzano \& Ocaña, 2020; } \\
\text { Yurkovetskiy et al., } 2021 .\end{array}$ \\
\hline MORAb-202 / Eisai Inc. & $\begin{array}{l}\text { Fungi/ADC (contains } \\
\text { farletuzumab and eribulin) }\end{array}$ & Solid tumours & $\begin{array}{l}\text { Humanized antibody farletuzumab against folate } \\
\text { receptor FR } \alpha \text {, beneficial load - eribulin MTA } \\
\text { (derivative of halichondrin B). Inhibitor of microtubules }\end{array}$ & $\begin{array}{l}\text { Cheng et al., 2018; } \\
\text { Matsunaga et al., } 2021 .\end{array}$ \\
\hline $\begin{array}{l}\text { Soblidotin (TZT-1027, } \\
\text { derivate Dolastatin 10)/ } \\
\text { Aska Pharmaceuticals }\end{array}$ & $\begin{array}{l}\text { Mollusks, cyanobacteria/ } \\
\text { Linear peptide }\end{array}$ & $\begin{array}{l}\text { Broad range of activity } \\
\text { against xenotransplantation } \\
\text { of human tumours }\end{array}$ & $\begin{array}{l}\text { Inhibits polymerization of tubulin, suspends division } \\
\text { of tumour cells in very low concentrations, suppresses } \\
\text { angiogenesis, inhibits processes of mytosis and } \\
\text { angiogenesis on the models of progressing tumour }\end{array}$ & $\begin{array}{l}\text { Akashi et al., 2007; } \\
\text { Martins et al., 2014; } \\
\text { Frau et al., 2019. }\end{array}$ \\
\hline $\begin{array}{l}\text { Plocabulin (PM 060184, } \\
\text { PM 184)/ Pharma Mar }\end{array}$ & Fungi / Polyketide & $\begin{array}{l}\text { Mammary gland tumours, } \\
\text { colorectal cancer, advanced } \\
\text { solid tumours }\end{array}$ & $\begin{array}{l}\text { Inhibits tubulin polymerization, processes of mytosis. } \\
\text { Strong inhibitor of microtubules with unique } \\
\text { molecular mechanism }\end{array}$ & $\begin{array}{l}\text { Newman \& Cragg, 2014; } \\
\text { Prota et al., 2014; Miller } \\
\text { et al., 2018; Pereira et al., } \\
\text { 2019; Jimenez et al., } 2020 .\end{array}$ \\
\hline
\end{tabular}




\begin{tabular}{|c|c|c|c|c|}
\hline $\begin{array}{c}\text { Name of drug/ } \\
\text { Company }\end{array}$ & $\begin{array}{l}\text { Marine organism/ } \\
\text { chemical class }\end{array}$ & Purpose, target & Mechanism of action & Reference \\
\hline $\begin{array}{l}\text { Zalypsis (PM 00104, } \\
\text { PM 10450)/ Pharma Mar }\end{array}$ & Mollusks / Alkaloid & $\begin{array}{l}\text { Cervical cancer, endomet- } \\
\text { rium cancer, Ewing's sarco- } \\
\text { ma, leukoses, lymphomas, } \\
\text { solid tumours, multiple } \\
\text { myeloma, urogenital cancer }\end{array}$ & $\begin{array}{l}\text { Inhibits proteins of cellular cycle, transcription factors; } \\
\text { DNA-binding protein modulator }\end{array}$ & $\begin{array}{l}\text { Newman \& Cragg, 2014; } \\
\text { Petek, 2014; } \\
\text { Ocio et al., 2016; } \\
\text { López-Iglesias, 2017; } \\
\text { Ruiz-Torres et al., } 2017 .\end{array}$ \\
\hline $\begin{array}{l}\text { Ladiratuzumab vedotin } \\
\text { (SGN-LIV1A, SLC39A6, ZIP6) } \\
\text { / Seatle Genetic Inc. }\end{array}$ & $\begin{array}{l}\text { Mollusks, cyanobacteria/ } \\
\text { ADC with MMAE }\end{array}$ & $\begin{array}{l}\text { Metastatic cancer of mam- } \\
\text { mary gland, melanoma, } \\
\text { prostate cancer, cancer of } \\
\text { the ovaries and the womb }\end{array}$ & $\begin{array}{l}\text { Aimed at transmembrane protein LIV-1 (SLC39A6). } \\
\text { Splits proteases, inhibits tubulin polymerase, mytosis } \\
\text { processes, increases apoptosis }\end{array}$ & $\begin{array}{l}\text { Boni et al., 2019; } \\
\text { Newman, 2019; } \\
\text { Pereira et al., } 2019 .\end{array}$ \\
\hline $\begin{array}{l}\text { Kahalalide F/ } \\
\text { PharmaMar, } \\
\text { Hawai University }\end{array}$ & $\begin{array}{l}\text { Mollusks, cyanobacteria/ } \\
\text { Cyclic dipeptide }\end{array}$ & $\begin{array}{l}\text { Cancers of mammary gland, } \\
\text { prostate, neuroblastoma, } \\
\text { osteosarcoma }\end{array}$ & $\begin{array}{l}\text { Inhibits expression of growth factor TGF- } \alpha \text {, signal } \\
\text { pathways of PI3K-AKT in cellular lines of mammary } \\
\text { cancer SKBR3 and BT474 }\end{array}$ & $\begin{array}{l}\text { Shilabin \& Hamann, 2011; } \\
\text { Martins et al., 2014; Leea et } \\
\text { al., 2017; Rosa et al., } 2019 .\end{array}$ \\
\hline RC-48 (Disitamab vedotin, $\mathrm{RC}$ & Various/Recombinatory & Mammary cancer, lung & Targets epidermal growth factor, receptory protein & Newman, 2019; \\
\hline 480-ADC) / RemGen & ADC antiHer2 with MMAE & $\begin{array}{l}\text { cancer, metastatic urothelial } \\
\text { cancer, stomach cancer }\end{array}$ & $\begin{array}{l}\text { tyrosine kinases erbB-2, EGFR2, HER2, HER-2, } \\
\text { p185c-erbB2, NEU,CD340 }\end{array}$ & $\begin{array}{l}\text { Li et al., 2020; } \\
\text { Sheng et al., } 2021 .\end{array}$ \\
\hline $\begin{array}{l}\text { CAB-ROR2-ADC (BA-3021, } \\
\text { CAB-AXL-ADC)/ BioAlta, } \\
\text { LLC }\end{array}$ & $\begin{array}{l}\text { Mollusks, cyanobacteria/ } \\
\text { ADC with } \mathrm{CAB} \text {, anti ROR- } \\
2 \text { with unknown cytotoxic } \\
\text { agent }\end{array}$ & $\begin{array}{l}\text { Sarcoma of the soft tissues, } \\
\text { non-small-cell lung cancer, } \\
\text { triple-negative breast cancer, } \\
\text { pancreatic cancer, melanoma }\end{array}$ & $\begin{array}{l}\text { Patetented technology. CAB-antibody selectively } \\
\text { binds with ROR2-expressing cancer cells, thereby } \\
\text { achieving maximum effect while having minimum } \\
\text { toxicity }\end{array}$ & $\begin{array}{l}\text { Newman, 2019; } \\
\text { Pereira et al., 2019; } \\
\text { Dyshlovoy \& Honecker, } \\
\text { 2020; Menck et al., 2021. }\end{array}$ \\
\hline $\begin{array}{l}\text { CX-2029/AbbVie, } \\
\text { CytomX Therapeutics }\end{array}$ & Various / ADC with MMAE & $\begin{array}{l}\text { Cancer of the head and neck, } \\
\text { non-small-cell lung cancer, } \\
\text { cancer of the pancreas, diffu- } \\
\text { sive large-cell lymphoma, } \\
\text { inoperable solid tumours, } \\
\text { B-cellular lymphomas } \\
\end{array}$ & $\begin{array}{l}\text { System uses Probody }{ }^{(\mathrm{IM})} \text { preparation aimed at CD71. } \\
\text { Has impact on cancer targets that are inaccessible for } \\
\text { regular ADC }\end{array}$ & $\begin{array}{l}\text { Polu \& Lowman, 2014; } \\
\text { Newman, 2019; } \\
\text { Autio et al., 2020. }\end{array}$ \\
\hline $\begin{array}{l}\text { Enapotamab vedotin } \\
\text { (HuMax-AXL-107)/ GenMab }\end{array}$ & $\begin{array}{l}\text { Mollusks / } \\
\text { Axl-specific ADC } \\
\text { with MMAE }\end{array}$ & $\begin{array}{l}\text { Ovary cancer, cervical } \\
\text { cancer, endometrial cancer, } \\
\text { pancreatic cancer, lung } \\
\text { cancer, melanoma }\end{array}$ & $\begin{array}{l}\text { Experimental chemoimmunoconjugate aimed at } \\
\text { receptory tyrosine kinase Axl, blocks polymerization } \\
\text { of tubulin in tumour cell, induces apoptosis of cancer } \\
\text { cells }\end{array}$ & $\begin{array}{l}\text { Koopman et al., 2019; } \\
\text { Newman, } 2019 .\end{array}$ \\
\hline $\begin{array}{l}\text { W0101 / } \\
\text { Pierre Fabre Medicament }\end{array}$ & $\begin{array}{l}\text { Mollusks, cyanobacteria/ } \\
\text { ADC with MMAE }\end{array}$ & Solid metastatic tumours & $\begin{array}{l}\text { Contains humanized antibody hz208F2-4, } \\
\text { specifically binds with receptor IGF-1R, blocks } \\
\text { polymerization of tubulin }\end{array}$ & $\begin{array}{l}\text { Akla et al., 2020; } \\
\text { Dyshlovoy \& Honecker, } \\
2020 .\end{array}$ \\
\hline \multicolumn{5}{|c|}{ Phase I } \\
\hline $\begin{array}{l}\text { ZW-49/ } \\
\text { Zymeworks Inc. }\end{array}$ & $\begin{array}{l}\text { Mollusks, cyanobacteria/ } \\
\text { Biospecific ADC with MMAE }\end{array}$ & $\begin{array}{l}\text { Metastatic tumours, } \\
\text { expressing Her } 2\end{array}$ & Aimed at Her2 on tumour cells & $\begin{array}{l}\text { Boni, 2020; } \\
\text { Tolcher, } 2020 .\end{array}$ \\
\hline ARX 788/Ambrx, Inc & $\begin{array}{l}\text { Mollusks, cyanobacteria/ } \\
\text { ADC with MMAE }\end{array}$ & $\begin{array}{l}\text { Her2-positive tumours of } \\
\text { the stomach, breast cancer }\end{array}$ & $\begin{array}{l}\text { Site-specific ADC of new generation, uses unique } \\
\text { technology of conjugation and non-splittable } \\
\text { Amberstatin (AS269). Active against tumour cells } \\
\text { with high expression of Her2. }\end{array}$ & $\begin{array}{l}\text { Skidmore et al., 2020; } \\
\text { Barok et al., } 2021 .\end{array}$ \\
\hline ALT-P7 / Alteogen, Inc. & $\begin{array}{l}\text { Mollusks, cyanobacteria/ } \\
\text { ADC with MMAE }\end{array}$ & Her2-positive breast cancer & Comprises trastuzumab biobetter HM2 and MMAE & $\begin{array}{l}\text { Rinnerthaler et al., 2019; } \\
\text { Luque-Bolivar et al., 2020; } \\
\text { Park et al., 2020. }\end{array}$ \\
\hline PF-06804103 / Pfizer & $\begin{array}{l}\text { Mollusks, cyanobacteria/ } \\
\text { ADC with Aur } 0101\end{array}$ & $\begin{array}{l}\text { Mammary gland cancer, } \\
\text { stomach cancer }\end{array}$ & $\begin{array}{l}\text { Variant of ADC which contains monoclonal } \\
\text { antibody anti-Her2, conjugated splittable linker with } \\
\text { cytotoxic agent Aur 0101. Aimed at Her2, }\end{array}$ & $\begin{array}{l}\text { Graziani et al., 2020; } \\
\text { Meric-Bernstam, } 2020 .\end{array}$ \\
\hline $\begin{array}{l}\text { PSMA-ADC / Seattle Genetics, } \\
\text { Roots Analysis, Progenics }\end{array}$ & $\begin{array}{l}\text { Mollusks, cyanobacteria/ } \\
\text { ADC with MMAE }\end{array}$ & $\begin{array}{l}\text { Cells of prostate cancer, } \\
\text { glioblastoma multiforme }\end{array}$ & $\begin{array}{l}\text { Intended for selective binding of PSMA-expressing } \\
\text { cells, inhibits proliferation of cells, nuining } \\
\text { cytoskeleton }\end{array}$ & $\begin{array}{l}\text { Wang et al., 2011; } \\
\text { Newman, 2019; } \\
\text { Petrylak et al., 2020. }\end{array}$ \\
\hline
\end{tabular}

Table 2

Anti-tumour substances, trials of which were completed at different phases of clinical studies

\begin{tabular}{|c|c|c|c|c|c|}
\hline $\begin{array}{l}\text { Name of drug / } \\
\text { Company }\end{array}$ & $\begin{array}{l}\text { Marine organism/ } \\
\text { chemical class }\end{array}$ & Purpose, target & Mechanism of action & References & $\begin{array}{l}\text { Phase } \\
\text { of study }\end{array}$ \\
\hline $\begin{array}{l}\text { Keyhole Limpet } \\
\text { Hemocyanin (KLH) / } \\
\text { Intracel, Rockville, } \\
\text { Immucothell }{ }^{\mathbb{}} \text {, Biosyn } \\
\text { Arzneimittel-GmbH, } \\
\text { Fellbach, Calbiochem }\end{array}$ & $\begin{array}{l}\text { Mollusks / } \\
\text { Glycoprotein }\end{array}$ & $\begin{array}{l}\text { Metastatic cancer of mammary gland, } \\
\text { bladder cancer, ovary cancer, cancers } \\
\text { of the pancreas, prostate, recurring } \\
\text { glioblastoma, neuroblastoma, non- } \\
\text { Hodgkin lymphoma, skin melanoma }\end{array}$ & $\begin{array}{l}\text { Modulates processes of synthesis of inflammatory } \\
\text { and pro-inflammatory mediators, increases early } \\
\text { and late apoptotic activity in MCF-7 cells, reduces } \\
\text { late apoptotic activity in ZR75-1 cells }\end{array}$ & $\begin{array}{l}\text { Lammers et al., 2012; } \\
\text { Wimmers et al., 2017; } \\
\text { Newman, 2019; } \\
\text { Román et al., 2019. }\end{array}$ & I/III \\
\hline $\begin{array}{l}\text { Iladatuzumab vedotin } \\
\text { (DCDS0780A, } \\
\text { RO7032005)/ } \\
\text { Hoffmann-La Roche }\end{array}$ & $\begin{array}{l}\text { Various / ADC } \\
\text { with MMAE }\end{array}$ & $\begin{array}{l}\text { Recurring or refractory B-cell } \\
\text { lymphoma }\end{array}$ & Targets CD-79A-cells & $\begin{array}{l}\text { Liersch et al., 2005; } \\
\text { Sahlmann et al., } 2017 .\end{array}$ & II \\
\hline $\begin{array}{l}\text { Indusatumab Vedotin } \\
\text { (MLN-0264, } \\
\text { 5F9vcMMAE, TAK- } \\
\text { 264)/ Seattle Genetics }\end{array}$ & $\begin{array}{l}\text { Mollusks, } \\
\text { cyanobacteria/ } \\
\text { ADC with MMAE }\end{array}$ & $\begin{array}{l}\text { Progressing pancreatic } \\
\text { adenocarcenoma, tumours of the } \\
\text { gastro-internal tract, which express } \\
\text { guanylate cyclase } 2 \mathrm{C}\end{array}$ & Aimed at guanylate cyclase $2 \mathrm{C}$ & $\begin{array}{l}\text { Newman \& Cragg, 2014; } \\
\text { Almhanna et al., 2017; } \\
\text { Gogineni \& Hamann, } \\
2018 .\end{array}$ & I/II \\
\hline $\begin{array}{l}\text { Pinatuzumab vedotin } \\
\text { (DCDT2980S, } \\
\text { FCU2703)/ } \\
\text { Genentech, Roche }\end{array}$ & $\begin{array}{l}\text { Mollusks, } \\
\text { cyanobacteria/ } \\
\text { ADC with MMAE }\end{array}$ & Non-Hodgkin lymphoma, leukosis & $\begin{array}{l}\text { Stimulates processes of apoptosis, inhibits } \\
\text { processes of mytosis, polymeresation of tubulin, } \\
\text { targets CD22 }\end{array}$ & $\begin{array}{l}\text { Forero-Torres et al., 2016; } \\
\text { Advani et al., 2017; } \\
\text { Morschhauser et al., 2019; } \\
\text { Yu et al., 2021. }\end{array}$ & I/II \\
\hline
\end{tabular}




\begin{tabular}{|c|c|c|c|c|c|}
\hline $\begin{array}{l}\text { Name of drug/ } \\
\text { Company }\end{array}$ & $\begin{array}{c}\text { Marine organism/ } \\
\text { chemical class }\end{array}$ & Purpose, target & Mechanism of action & References & $\begin{array}{l}\text { Phase } \\
\text { of study }\end{array}$ \\
\hline $\begin{array}{l}\text { Glembatumumab } \\
\text { vedotin(CDX-011, } \\
\text { CR011)/Seatle } \\
\text { Genetics, Celldex } \\
\text { Therapeutics }\end{array}$ & $\begin{array}{l}\text { Mollusks, } \\
\text { cyanobacteria / } \\
\text { ADC with MMAE }\end{array}$ & $\begin{array}{l}\text { Squamous cell lung cancer, } \\
\text { melanoma, triple negative cancer of } \\
\text { the mammary gland, recurring } \\
\text { osteocarcinoma, uveal melanoma }\end{array}$ & $\begin{array}{l}\text { Induces partial or complete regression of cancer } \\
\text { cells that express GPNMB; inhibits the set of } \\
\text { microtubules }\end{array}$ & $\begin{array}{l}\text { Ott et al., 2019; Patrick et al., } \\
\text { 2019; Pereira et al., 2019; } \\
\text { Wolska-Washer \& Robak, } \\
\text { 2019; Hasanov et al., 2020; } \\
\text { Kaštelan et al., 2020. }\end{array}$ & I/II \\
\hline $\begin{array}{l}\text { Tasidotin (ILX-651, } \\
\text { derivate Dolastatin 15) / } \\
\text { Genzyme Corporation }\end{array}$ & $\begin{array}{l}\text { Mollusks / } \\
\text { Pentapeptide }\end{array}$ & $\begin{array}{l}\text { Erythroid leukosis, ovary cancer, } \\
\text { carcinoma of the large intestine, cells } \\
\text { of mammary cancer, lung carcinoma, } \\
\text { melanoma }\end{array}$ & $\begin{array}{l}\text { Inhibits processes of proliferation, mytosis, } \\
\text { polymerization of tubulin in microtubules }\end{array}$ & $\begin{array}{l}\text { Ray et al., 2007; } \\
\text { Martins et al., 2014; } \\
\text { Frau et al., } 2019 .\end{array}$ & I/II \\
\hline $\begin{array}{l}\text { PF-06263507 (A1- } \\
\text { mcMMAF)/ Pfizer }\end{array}$ & $\begin{array}{l}\text { Mollusks, } \\
\text { cyanobacteria/ } \\
\text { ADC with MMAF }\end{array}$ & Progressing solid tumours, leukoses & $\begin{array}{l}\text { Fragment of antibody selectively binds with 5T4- } \\
\text { expressing cells. Inhibits polymerization of tubulin, } \\
\text { leads to suspension of phase G2 / M and apoptosis } \\
\text { of tumour cells }\end{array}$ & $\begin{array}{l}\text { Forero-Torres et al., 2016; } \\
\text { Shapiro et al., 2017; } \\
\text { Gogineni \& Hamann 2018; } \\
\text { Newman, 2019. }\end{array}$ & I \\
\hline $\begin{array}{l}\text { DMUC-5754A(Sofi- } \\
\text { tuzumab vedotin, RG- } \\
\text { 7458)/Hoffmann-La } \\
\text { Roche Ltd, Genentech }\end{array}$ & $\begin{array}{l}\text { Mollusks, } \\
\text { cyanobacteria/ } \\
\text { ADC with MMAE }\end{array}$ & Ovary and pancreatic cancers & $\begin{array}{l}\text { Contains humanitized monoclonal antibody against } \\
\text { MUC16. Inhibitor of tubulin polymerase, apoptosis } \\
\text { stimulator }\end{array}$ & $\begin{array}{l}\text { Newman \& Cragg, 2014; } \\
\text { Liu et al., 2016; } \\
\text { Gogineni \& Hamann, } 2018 .\end{array}$ & I \\
\hline $\begin{array}{l}\text { Elisidepsin (PM02734, } \\
\text { Irvalec; 15b)/ Pharma } \\
\text { Mar }\end{array}$ & $\begin{array}{l}\text { Mollusk/ } \\
\text { Depsipeptide }\end{array}$ & $\begin{array}{l}\text { Solid tumours, metastatic cancer of } \\
\text { the esophagus, stomach cancer, } \\
\text { squamous cell lung cancer }\end{array}$ & $\begin{array}{l}\text { Self-organizes and modifies lipid constituents of } \\
\text { plasmatic membrane of tumour cells, increases } \\
\text { penetrability of cells, induces apoptosis }\end{array}$ & $\begin{array}{l}\text { Petty et al., 2016; Kang et al., } \\
\text { 2018; Van Andel et al., 2018; } \\
\text { Chakraborty \& Joy, } 2020 .\end{array}$ & $\mathrm{I}$ \\
\hline $\begin{array}{l}\text { Bryostatin-1/ } \\
\text { National Cancer } \\
\text { Institute (USA) }\end{array}$ & $\begin{array}{l}\text { Bryozoa/ } \\
\text { Macrocyclic } \\
\text { lactone }\end{array}$ & $\begin{array}{l}\text { Melanoma, lymphoma, ovary } \\
\text { carcinoma, liver carcinoma, leukosis. } \\
\text { Orphan drug in combination with } \\
\text { paclitaxel during esophagus cancer }\end{array}$ & $\begin{array}{l}\text { Selective inhibitor of non-typic isophorm of protein } \\
\text { kinese C. Induces synthesis of cytotoxins, immune- } \\
\text { stimulator }\end{array}$ & $\begin{array}{l}\text { Popov, 2006; } \\
\text { Kim \& Guan, 2015; } \\
\text { Ciavatta et al., 2020; } \\
\text { Raghuvanshi et al., } 2020 .\end{array}$ & I \\
\hline $\begin{array}{l}\text { DNIB-0600A } \\
\text { (Lifastuzumab vedotin, } \\
\text { RG-7599, GTPL8405)/ } \\
\text { Genentech Inc. }\end{array}$ & $\begin{array}{l}\text { Mollusks, } \\
\text { cyanobacteria/ } \\
\text { ADC with MMAE }\end{array}$ & $\begin{array}{l}\text { Non-small-cell lung cancer, platinum- } \\
\text { resistant ovarian cancer }\end{array}$ & $\begin{array}{l}\text { Targets NaPi2b epitope, blocks polymerization of } \\
\text { tubulin }\end{array}$ & $\begin{array}{l}\text { Newman \& Cragg, 2017; } \\
\text { Gogineni \& Hamann, } \\
\text { 2018; Gerber et al., 2020; } \\
\text { Moore et al., 2020. }\end{array}$ & I \\
\hline $\begin{array}{l}\text { DMOT-4039A } \\
\text { (RG-7600)/ } \\
\text { Genentech Inc. }\end{array}$ & $\begin{array}{l}\text { Mollusks, } \\
\text { cyanobacteria/ } \\
\text { ADC with MMAE }\end{array}$ & $\begin{array}{l}\text { Inoperable pancreatic or platinum- } \\
\text { resistant ovarian cancer }\end{array}$ & $\begin{array}{l}\text { Contains humanitized IgG1-anti mesothelin mAb } \\
\text { h7D9.v3. Destroys the microtubule network, } \\
\text { induces apoptosis }\end{array}$ & $\begin{array}{l}\text { Newman \& Cragg, 2014; } \\
\text { Weekes et al., 2016; Go- } \\
\text { gineni \& Hamann, } 2018 .\end{array}$ & $\mathrm{I}$ \\
\hline $\begin{array}{l}\text { DEDN-6526A(RG- } \\
\text { 7636)/ Genentech, } \\
\text { Seattle Genetics }\end{array}$ & $\begin{array}{l}\text { Mollusks, } \\
\text { cyanobacteria/ } \\
\text { ADC with MMAE }\end{array}$ & Inoperable melanoma & $\begin{array}{l}\text { Anti-microtubule agent, inhibitor of tubuline- } \\
\text { polymerase, mitosis, apoptosis inducer }\end{array}$ & $\begin{array}{l}\text { Newman \& Cragg, 2014; } \\
\text { Gogineni \& Hamann, 2018; } \\
\text { Nejadmoghaddam et al., } \\
2019 .\end{array}$ & $\mathrm{I}$ \\
\hline $\begin{array}{l}\text { Vandortuzumab vedotin } \\
\text { (DSTP-3086S, RG- } \\
\text { 7450)/Genentech } \\
\text { (Roche) }\end{array}$ & $\begin{array}{l}\text { Mollusks, } \\
\text { cyanobacteria/ } \\
\text { ADC with MMAE }\end{array}$ & Metastatic prostate cancer & $\begin{array}{l}\text { Modified humanitized antibody against STEAP1 } \\
\text { IgG1, bond with MMAE through Mc-VC-PABC. } \\
\text { Aims at epithelial prostate antigene }\end{array}$ & $\begin{array}{l}\text { Newman \& Cragg, 2014; } \\
\text { Van Andel et al., } 2018 .\end{array}$ & $\mathrm{I}$ \\
\hline $\begin{array}{l}\text { Sirtratumab vedotin } \\
\text { (AGS-15E, AGS- } \\
\text { 15ME)/Agensys, } \\
\text { Seattle Genetics }\end{array}$ & $\begin{array}{l}\text { Mollusks, } \\
\text { cyanobacteria/ } \\
\text { ADC with MMAE }\end{array}$ & $\begin{array}{l}\text { Cancers of the bladder, lungs, } \\
\text { mammary glands, glioblastoma }\end{array}$ & $\begin{array}{l}\text { The first and the only agent aimed at } \\
\text { transmembrane protein SLITRK6. Effectively } \\
\text { binds with target cells, induces apoptosis }\end{array}$ & $\begin{array}{l}\text { Hofland, 2016; Petrylak et } \\
\text { al., 2016; Pereira et al, } \\
2019 .\end{array}$ & $\mathrm{I}$ \\
\hline $\begin{array}{l}\text { AGS 67E/Agensys, } \\
\text { Inc., Astellas Pharma }\end{array}$ & $\begin{array}{l}\text { Mollusks, } \\
\text { cyanobacteria/ } \\
\text { ADC with MMAE }\end{array}$ & $\begin{array}{l}\text { Aims at CD37. Refractory } \\
\text { lymphocytic leukemia, non-Hodgkin } \\
\text { lymphoma }\end{array}$ & $\begin{array}{l}\text { Targeted at CD37. Has high cytotoxicity, induces } \\
\text { apoptosis and changes in cellular cycle in many } \\
\text { cellular lines }\end{array}$ & $\begin{array}{l}\text { Pereira et al., 2015; } \\
\text { Newman et al, 2017; } \\
\text { Pereira et al., 2019. }\end{array}$ & $\mathrm{I}$ \\
\hline $\begin{array}{l}\text { Cofetuzumab pelidotin } \\
\text { (PF-06647020)/ Pfizer }\end{array}$ & $\begin{array}{l}\text { Mollusks / ADC } \\
\text { with auristatin } \\
\text { (anti-PKT7) }\end{array}$ & Solid tumours & $\begin{array}{l}\text { Mitosis inhibitor, apoptosis stimulator, inhibitor of } \\
\text { tubuline polymerisation }\end{array}$ & $\begin{array}{l}\text { Katoh, 2017; } \\
\text { Newman, } 2019 .\end{array}$ & $\mathrm{I}$ \\
\hline $\begin{array}{l}\text { PF-06380101 } \\
\text { (Q8020AX34E)/ Pfizer }\end{array}$ & $\begin{array}{l}\text { Mollusks, } \\
\text { cyanobacteria/ } \\
\text { ADC with MMAE }\end{array}$ & $\begin{array}{l}\text { Solid tumours, triple negative and } \\
\text { metastatic breast cancer }\end{array}$ & $\begin{array}{l}\text { Analogue of dolastatin 10, inhibitor of } \\
\text { polymerization of tubulin }\end{array}$ & $\begin{array}{l}\text { Rago et al., 2017; } \\
\text { Pereira et al., } 2019 .\end{array}$ & I \\
\hline $\begin{array}{l}\text { ABBV-085 } \\
\text { (Samrotamab vedotin)/ } \\
\text { AbbVie, Inc. }\end{array}$ & $\begin{array}{l}\text { Various / Anti- } \\
\text { huLRRC15 ADC }\end{array}$ & $\begin{array}{l}\text { Solid tumours, undifferentiated } \\
\text { pleoform sarcoma, squamous cell } \\
\text { cancer of the head and neck, } \\
\text { mammary gland cancer }\end{array}$ & $\begin{array}{l}\text { Aims at LRRC15 in the micro-environment of } \\
\text { tumour }\end{array}$ & $\begin{array}{l}\text { Maderna et al., 2014; } \\
\text { Purcell et al., 2018; } \\
\text { Pereira et al., 2019, } \\
\text { Hingorani et al., 2021. }\end{array}$ & I \\
\hline $\begin{array}{l}\text { XMT 1522 (TAK-522) } \\
\text { / Mersana Therapeutics }\end{array}$ & $\begin{array}{l}\text { Various/ADC with } \\
\text { MMAF-HPA }\end{array}$ & $\begin{array}{l}\text { Cancers of the stomach, lungs, } \\
\text { mammary glands }\end{array}$ & Anti-Her2-antibody-medical preparation & $\begin{array}{l}\text { Hamilton et al., 2018; Le } \\
\text { Joncour et al., } 2019 .\end{array}$ & $\mathrm{I}$ \\
\hline $\begin{array}{l}\text { Hemiasterlin E7974/ } \\
\text { Eisai Inc. }\end{array}$ & $\begin{array}{l}\text { Fungi/Linear } \\
\text { tripeptide }\end{array}$ & Solid tumours & $\begin{array}{l}\text { In vitro inhibitor of polymerization of tubulin, pro- } \\
\text { liferation of broad range of cancer cells, overcomes } \\
\text { the resistance against other anti-tubulin agents }\end{array}$ & $\begin{array}{l}\text { Rocha-Lima et al., 2012; } \\
\text { Marchetti et al., 2016; Won et } \\
\text { al., 2019; Zhang et al., 2021. }\end{array}$ & I \\
\hline $\begin{array}{l}\text { BAY 79-4620 } \\
\text { (3ee9MMAE)/ Seattle } \\
\text { Genetics }\end{array}$ & $\begin{array}{l}\text { Mollusks, } \\
\text { cyanobacteria/ } \\
\text { ADC with MMAE }\end{array}$ & $\begin{array}{l}\text { Progressing solid tumours, CA9- } \\
\text { positive stomach cancer }\end{array}$ & $\begin{array}{l}\text { Targets carbonic anhydrase IX(CA9) of human, } \\
\text { inhibitor of tubulin polymerization }\end{array}$ & $\begin{array}{l}\text { Francisco et al., 2003; } \\
\text { Petrul et al., } 2012\end{array}$ & I \\
\hline $\begin{array}{l}\text { Denintuzumab ma- } \\
\text { fodotin (SGN-CD19A, } \\
\text { SGN-19A/BU12-491) } \\
\text { / Seattle Genetics }\end{array}$ & $\begin{array}{l}\text { Mollusks, } \\
\text { cyanobacteria/ } \\
\text { ADC with MMAF }\end{array}$ & $\begin{array}{l}\text { CD19-positive acute lymphoblastic } \\
\text { leukemia, B-cell non-Hodgkin } \\
\text { lymphoma }\end{array}$ & $\begin{array}{l}\text { Humanitized anti-CD19-antibody, } \\
\text { anti-microtubular agent }\end{array}$ & $\begin{array}{l}\text { Shah et al., 2017; Jones et } \\
\text { al., 2019; Newman. 2019; } \\
\text { Pereira et al., 2019; Mc } \\
\text { Nerney \& Teachey, } 2021 .\end{array}$ & $\mathrm{I}$ \\
\hline $\begin{array}{l}\text { SGN-75 (Vorse- } \\
\text { tuzumab mafodotin)/ } \\
\text { Seattle Genetics }\end{array}$ & $\begin{array}{l}\text { Mollusks, } \\
\text { cyanobacteria/ } \\
\text { ADC with MMAF }\end{array}$ & $\begin{array}{l}\text { Recurring and refractory non- } \\
\text { Hodgkin lymphoma, hepatocellular } \\
\text { adenocarcinoma }\end{array}$ & $\begin{array}{l}\text { Induces apoptosis of tumour cells, inhibits } \\
\text { proliferation of tumour cells that overexpress } \\
\text { CD70 }\end{array}$ & $\begin{array}{l}\text { Tannir at al., 2014; } \\
\text { Pereira et al., } 2019\end{array}$ & I \\
\hline
\end{tabular}




\section{Trials of biopharmaceutic production}

Preparation of a pharmaceutical product starting from the moment of discovering the biochemical potential of a substance to the industrial production of drug includes laboratory, pre-clinical and clinical trials. The pharmaceutical company determines the chemical and molecular formula of the preparation, develops the form of the production, and then conducts pre-clinical trials. The pre-clinical stage includes many biological, microbiological, pharmacological, chemical, physical and toxicological studies on cellular lines or laboratory animals. The main goal of pre-clinical trials of preparation is obtaining evidences of the efficacy and safety of the preparation. By the end of pre-clinical trials of preparation, its action is tested in at least three stages on people who gave their voluntary consent. Preparation goes to the next stage only after it has proved to demonstrate satisfactory results in the previous one.

Clinical trials at phase I are carried out on 10-15 healthy volunteers or terminally ill patients with their agreement. The goal of the trials is determining the pharmacokinetic and pharmacodynamic parameters, parameters of toxicity, preliminary evaluation of safety of and tolerance to the preparation. Also, the trials examine the parameters of absorption, processes of metabolism, distribution and excretion in the organism, and determine the preferable form of application and safe level of dose. Clinical surveys of the first phase last several weeks to one year.

The goals of trials at phase II entail determination of optimum dosage and selecting the scheme of introduction of the preparation. At this stage, the number of tested patients increases up to 300 patients, the details of dosages, mechanisms of action, side-effects and the methods of their minimization are tested.

Clinical trials at phase III are randomizing controllable multi-center surveys involving a large group of volunteer patients $-1,000$ and more. The goals of phase III are to confirm the preliminary data obtained during the previous studies and compare the actions of the preparations to standard therapy regarding particular oncologic disease. At this stage, therapeutic efficiency of the preparation is studied depending on the dose. If, by the end of the clinical studies at phase III, the positive effect of treatment remains, the patients continue to receive this drug until stable remission. Surveys at phase III may be continued if the sponsoring company wishes to broaden the drug's to-use indications, and are classified as phase IIIB.

Interestingly, in cancer patients with late stages of diseases, antitumour natural compounds tested in randomized conditions as monopreparations or combined with other chemotherapeutic preparations demonstrated longer clinical response compared with the traditional chemopreparations (Blay et al., 2015; El Bairi et al., 2017; Teplinsky \& Herzog, 2017).

Phase IV consists of post-registration clinical trials. They are aimed at collecting data on certain criteria: treatment periods, interaction of new preparation with other medical preparations or food products; analysis of use among patients of various age groups, economic parameters, remote results of treatment, and also collecting additional data on the safety and efficacy of the preparation on the basis of large groups of patients over a long period. During the trials, there may occur a problem of high toxicity and medical resistance, and therefore the evaluation of the effectiveness of clinical surveys of preparations on people often remains limited (Eastman, 2017; El Bairi et al., 2017). The process of preparing a pharmaceutic preparation lasts $10-15$ years and costs millions of dollars, and at the same time, less than $12 \%$ of potential drugs are ultimately allowed for use. If, over the cource of clinical studies, rare but dangerous unsatisfactory processes are found, the preparation is withdrawn from sale.

Scientists who are developing natural marine medical products isolate only several milligrams of promising compounds of new structure. Preliminary in vitro screening on the cancer cell lines requires a small amount of pure compounds. Studies on animals require around $100 \mathrm{mg}$, and clinical trials - up to several hundreds of grams, but kilograms are needed for its production as a pharmaceutical preparation (Newman, 2016). Biologically active substances from marine organisms are not always available in the required amount. Therefore, for many products obtained from hydrobionts, the only possible way to solve this problem is believed to be chemical synthesis. This method, other than production of the necessary amount of a certain substance, gives an additional advantage - obtaining valuable intermediate compounds and their analogues, which would later be used in further studies. Chemical synthesis also includes the development of expensive derivatives with more manageable, less complex, properties. For example, production of kahalalide $\mathrm{F}$ for clinical trials was developed using the methods of chemistry of solid-phase peptide synthesis, resulting in preparation of over 150 its analogues (Gao \& Hamann, 2011). Synthetic methods developed to obtain dolastatins led to emergence of new intermediate substances, such as auristatins, broadly used in contemporary antibody-drug conjugates (Cragg et al., 2012; Newman \& Cragg, 2014; Newman \& Cragg, 2017). Use of new technologies of aquaculture and profound knowledge in the sphere of genomics and biotechnology allow a synthesis of analogues of natural marine products through chemical synthesis on industrial scales.

\section{Perspectives of using cytotoxic compounds of marine origin in oncology and new biomedical technologies}

A great role in the emergence and development of tumour diseases is played by infectious agents (Pimenoff et al., 2019). In 2018, there were diagnozed 2.2 $\mathrm{M}$ cases of oncological diseases associated with infectious origin, which accounted for almost $13 \%$ of all cases of cancer (De Martel et al., 2020). There is an increasing number of studies confirming high risk of infection and severe course of COVID-19 in cancer patients (He et al., 2021; Li et al., 2021; Tian et al., 2021). Therefore, there is a sharp need to combat infectious triggers of carcenogenesis, which would allow a decrease in the level of morbidity and mortality. Cytotoxic substances of marine organisms, having unique chemical structure, anti-cancer, antiviral, immune-modulating, neuro-protective, antibacterial and many other effects, can make a systemic multi-target impact on cancer cells, as well as infectious agents (Fitton et al., 2020; Gentile et al., 2021; TaglialatelaScafati, 2021). Accordingly, a drug of marine origin Plitidepsin (Apli$\operatorname{din}^{B}$ ), used to treat tumour diseases, in in vitro studies displayed 27.5 times the antiviral activity of remdesivir, the only officially FDA-approved antiviral preparation to treat patients with SARS-Cov-2, and had lower toxicity (White et al., 2021). Studies revealed that along with Plitidepsin, such preparations as Eribulin mesylate and Trabectedin, having properties of inhibitors of SARS-Cov-2 protease, significantly reduce replication of the virus, and therefore the extent of severity of viral infection in cancer patients (Kalhotra et al., 2021). The marine organisms are known to produce antiviral compounds against many other pathogenic viruses, such as HIV, HSV, HHV, influenza A, smallpox virus, SRV-2, HAV, HBV, HCV; EBV, enterovirus, HCMV, JEV, TMV, PrV, WSSV, MS2, CHIKV, OsHV, SINV, TBE, PRRSV, EV-71, FIPV, MHV, BVDV, KHV, WEEV). Nonetheless, there is currently known only one compound of marine origin with antiviral activity against virus of herpes simplex and Varicella zoster - Vidarabin, used in medicine, and another one - Griffithsin, which is undergoing clinical tests against HIV (Riccio et al., 2020).

Drugs of marine origin are used to treat patients with bronchial asthma, Alzheimer's disease, hypertonic disease, with disorders in the processes of blood circulation, autoimmune diseases, pain syndromes, etc. Other than their direct purpose, they may be used for therapy with combined pathology in cancer patients, which would likely reduce drug load on patients, significantly optimize time and financial costs on conducting preclinical and clinical trials at early phases.

During the tests, it was found that many bioactive substances of marine origin have additive and synergic effects when combined with traditional anti-tumour preparations. Therefore, Trabectedin (marine alkaloid isolated from Ecteinascidia turbinata tunicate), by functioning as a DNA intercalator, coupled with vincristine increases therapeutic effect during treatment of sarcomas of the soft tissues. This allows doses of applied antitumour drugs to be decreased, thereby decreasing their toxicity and improving therapeutic index. Likewise, combination therapy may prevent drug-resistance, which significantly limits the traditional anti-tumour medical preparations (Calcabrini et al., 2017).

Contemporary medicine is oriented at application of target methods of cancer therapy, including molecular, cellular, gene therapies and preventive measures, antibody-drug conjugates, nanoparticles of biopolymers, metals, polymeric micelles and other nanoparticles (Liu et al., 2021). Antibody-drug conjugates are a separate class of immune-chemotherapy preparations. Substances isolated from marine organisms (or their synthe- 
tic analogues) are considered to be promising beneficial loads for future ADC: hemiasterlin, taltobulin, cemadotin, cryptoficine, discodermolide (Ponziani et al., 2020). New knowledge of the carcenogenesis processes and successes of gene engineering led to development of bispecific antibodies (bsAbs) that have an effect on two targets of carcenogenesis - alter the processes of cellular signal transmission and suspend further growth and metastasis of tumours (Liu et al., 2021). Unlike regular chemotherapeutic dugs, antibody-drug conjugates are a promising and most dynamically developing class of drugs, and have broad therapeutic range due to effective and specific transport of drug to antigen-expressing tumour cells (Mukherjee et al., 2019; Khongorzul et al, 2020). The FDA currently allow nine of them to be used, and over 80 of them, which are aimed at over 50 various antibodies, are at different phases of 600 clinical trials (Boni, et al., 2020, Barok et al., 2021). Despite the high efficiency and promising character of ADCs of the last generation, their synthesis is associated with certain difficulties such as humanization of antibodies, complexity of the processes of modification and conjugation with beneficial load. A number of studies suggest solving these problems by using aptamers, siRNA and DNA-lygands in the content of the conjugants (ApTDC, ASO) (Roberts, 2020). Unlike antibodies, aptamers require no humanization, are easily synthesized and modifiable. In a recent study, E3 aptamer-drug conjugates with beneficial loads MMAE and MMAF (derivatives of marine peptide dolastatin 10) exerted high anti-tumour activity towards many lines of tumour cells (Gray et al., 2020). To improve pharmacokinetics, bioactivity and rates of release of oncological preparations, there are studies underway oriented at developing ADCs of next generation, using site-specific conjugation, changes in the amount and position of beneficial loads, separate particles of completely humanized antibodies.

Numerous studies are currently being conducted dealing with the efficiency of cancer gene therapy, $60 \%$ of studies in the sphere of genetic medicine are conducted specifically in oncology (Stuijvenberga et al., 2020). As known, mutations of somatic cells are essential in emergence of tumours and development of medical resistance. Therefore, one of the promising directions in oncology is considered to be gene therapy. Genome DNA is the main target for natural anti-tumour therapeutic preparations (Van Stuijvenberg, 2020). Development of genetic technologies also led to the understanding of critical importance of RNA-influenced regulation of carcinogenesis in conditions of multiple tumours. Thus, the technology of RNA-influenced gene inhibition is considered promising, which demonstrated high efficiency in trials on multiple malignant tumours. RNA-therapeutic technologies in combination with immune- and chemotherapy inhibit several pathways of carcinogenesis at the same time, including overexpression of genes that modulates immune-enzymic system, thus arresting the growth of tumours, process of metastasis and overcoming chemo-resistance (Khan et al., 2021). Gene therapy of cancer involves use of viruses, immune-liposomes that contain stem cells, including autological cells, and also antibodies, nanoparticles of bioactive compounds. At the same time, activation of the pro-drug occurs directly in tumour cells, thereby preventing systemic complications. As active agents, gene therapy successfully uses preparations from marine organisms. Therefore, high efficacy at phase I of the trials was demonstrated by combined use of gemcitabine and siG12D-LODE ${ }^{\mathrm{TM}}$ (miniature biodegradable implant based on small interfering RNA, which is aimed specifically at KRAS oncogene) to treat locally advanced pancreatic cancer (Golan et al., 2015). Chitosan and its modification are broadly used as a non-viral vector of introduction of biomacromolecules and low-molecular medical preparations in gene therapy of cancer and potential immune adjuvant for antitumour vaccines (Babu \& Ramesh, 2017; Ye et al., 2019). Moreover, combination of analytical and genome approaches allowed prediction and synthesis of hypothetic analogues from a limited bioresource of natural products, which have new properties, differing from the initial (Smith et al., 2018). Cancer tumours are accompanied by chronic inflammation and synthesize a number of signal molecules, preparing grounds for their own development and formation of metastatic niches that increasingly colonize a large space. Therefore, some of the most promising directions in cancer therapy would be the methods oriented at cancer signal molecules, effector cells, system of mediators of chronic inflammation, microenvironment of tumours, metastatic niches. The effectiveness and tolerability of some anti-tumour preparations are known to depend on the geno- type of the patient, and therefore in the future, anti-tumour therapy will be of personalized pattern with consideration of the genotype peculiarities of the person.

Nanotechnologies are actively used in ongoing work on developing multi-modal systems to diagnose tumour diseases and transport preparations using hybrid particles based on biocompatible polymers that contain magnetic nanoparticles, photoacustic, fluorescent and medical components (Novoselova et al., 2021). To transport drugs to tumours, biodegradable nanopolymer particles of marine origin are actively being used: chitosan, carrageenan, alginate, fucoidan (Gagliardi et al., 2021). Chitosan is a universal cation polysaccharide of marine origin, which was approved by the FDA as a nano-carrier of medical preparations. It is used in nanomedicine due to its unique cation properties, non-immunogenity, biodegradation and biocompatibility (Islam et al., 2017; Al-Musawi et al., 2020; Islam et al., 2020). Carrageenan is a sulfated polysaccharide from brown algae, has a low potential to produce nanoparticles, but at the same time leads to prolonged release of medical agent from the epithelial and mucous tissues (George et al., 2019). Alginate is a linear anion polymer obtained from brown algae, is well soluable in physiologic fluids, interacts with various bioactive substances as a nanocarrier. Alginate nanoparticles are obtained using different methods, allowing one to combine them with various materials, for example nanoparticles based on chitosan, chemopreparations doxorubicin, 5-fluorouracil (Boekhoven et al., 2015), use in technologies with addition of superparamagnetic particles of iron oxide (Ma et al., 2008; Wang et al., 2017), in tissue engineering (Singh et al., 2020). Fucoidan is a sulfated biopolysacharide from brown marine algae, which has a broad range of biological, including anti-tumour, activities (Fitton et al., 2019), plays a great role in development of nano- and microparticles, is used as a nanocarrier and drug agent (Hsu et al., 2019; Wang et al., 2019). As nanocarriers of drugs, ulvan and laminarin, obtained from brown algae are also being studied. All mentioned polysaccharides are studied in the field of nanomedicine not only as a system of transport of drugs to tumours, but also as drugs because of the many effects they exert: induction of apoptosis, regulation of signal transduction pathways, inhibition of migration and angiogenesis, modulation of immunity and antioxidant system (Zhong et al., 2020). Aside from targeted impact on tumour tissues, natural nanoparticles with chemotherapeutic load are able to overcome natural biological barriers, thereby coming to the organism's hardest places to reach (Tan et al., 2020). The greatest advantages of natural nanocarriers are non-toxicity, biocompatibility and biodegradability, which make them more promising for development of new methods of combating oncological diseases. Thus, natural compounds from marine organisms are studied and used as monopreparations, nanocarriers, chemotherapy loads, molecular vectors for transport of cytotoxic loads to the tumour tissues (Tursunuva et al., 2018; Ou et al., 2020; Subjakova et al., 2021).

\section{Conclusions}

Medical resistance, genetic mutation, and population growth contribute to the emergence of new pathogens that cause diseases which were unknown earlier. This dictates the necessity of development of new drugs and improvement of the existing ones. The review presents the most recent data on contemporary biologically active compounds of marine origin, their value in practical medicine, biomedical and pharmaceutical spheres. Annually, 1,000 new molecules are being isolated from marine organisms, many of which have complex molecular structure, contain many potentially bioactive groups with so far undetermined mechanism of action (Van Andel et al., 2018). Marine organisms are used not only to obtain biologically active combinations, but in some cases as experimental models with unique experimental properties that provide additional possibilities for modeling and studying the biology of cancer (Elliot et al., 2020). Active interaction between representatives of various directions of science, medicine and industry would allow an effective implementation of all the stages of obtaining the final pharmaceutical product from marine organisms starting from the moment of discovery to use in practice identification of combinations, determining their biological activity, chemical synthesis, development of methods of targeted transport, clinical trials, to introduction into production (Newman, 2016; Barreca et al., 2020). 
This work was carried out within the framework of the government research assignment of IBSS RAS "Investigation of the mechanisms of controlling production processes in biotechnological complexes with the aim of developing the scientific foundations for the production of biologically active substances and technical products of marine genesis" No 121030300149-0. This study was carried out within the framework of a State Assignment No. 121051100109-1 of IBIW RAS, and No. AAAAA16-116090850007-7 of FSBEI HE Yaroslavl SAA

\section{The authors claim no conflict of interests.}

\section{References}

Abidizadegan, M., Peltomaa, E., \& Blomster, J. (2021). The potential of cryptophyte algae in biomedical and pharmaceutical applications. Frontiers in Pharmacology, 11,618836 .

Advani, R. H., Lebovic, D., Chen, A., Brunvand, M., Goy, A., Chang, J., Hochberg, E., Yalamanchili, S., Kahn, R., Lu, D., Agarwal, P., Dere, R. C., Hsieh, H.-J., Jones, S., Chu, Y.-W., \& Cheson, B. D. (2017). Phase I study of the anti-CD22 antibody-drug conjugate pinatuzumab vedotin with/without rituximab in patients with relapsed/refractory B-cell non-Hodgkin's lymphoma. Clinical Cancer Research, 23(5), 1167-1176.

Agrawal, S., Adholeya, A., \& Deshmukh, S. K. (2016) The pharmacological potential of non-ribosomal peptides from marine sponge and tunicates. Frontiers in Pharmacology, 7,333.

Ahmad, T. B., Liu, L., Kotiw, M., \& Benkendorff, K. (2018). Review of anti-inflammatory, immune-modulatory and wound healing properties of molluscs. Journal of Ethnopharmacology, 210, 156-178.

Aiub, C., Giannerini, A., Ferreira, F., Mazzei, J., Stankevicins, L., Lobo-Hajdu, G., Guimaraes, P., Hajdu, E., \& Felzenszwalb, I. (2006). Genotoxic evaluation of extracts from Aplysina fulva, a brazilian marine sponge. Mutation Research, $611,34-41$.

Akashi, Y., Okamoto, I., Suzuki, M., Tamura, K., Iwasa, T., Hisada, S., Satoh, T., Nakagawa, K., Ono, K., \& Fukuoka, M. (2007). The novel microtubule-interfering agent TZT-1027 enhances the anticancer effect of radiation in vitro and in vivo. British Journal of Cancer, 96(10), 1532-1539.

Akla, B., Broussas, M., Loukili, N., Robert, A., Beau-Larvor, C., Malissard, M., Boute, N., Champion, T., Haeuw, J.-F., Beck, A., Perez, M., Dreyfus, C., Pavlyuk, M., Chetaille, E., \& Corvaia, N. (2020). Efficacy of the antibody-drug conjugate W0101 in preclinical models of IGF-1 receptor overexpressing solid tumors. Molecular Cancer Therapeutics, 19(1), 168-177.

Almhanna, K., Wright, D., Mercade, T. M., Van Laethem, J.-L., Gracian, A. C., Guillen-Ponce, C., Faris, J., Lopez, C. M., Hubner, R. A., Bendell, J., Bols, A., Feliu, J., Starling, N., Enzinger, P., Mahalingham, D., Messersmith, W., Yang, H., Fasanmade, A., Danaee, H., \& Kalebic, T. (2017). A phase II study of antibody-drug conjugate, TAK-264 (MLN0264) in previously treated patients with advanced or metastatic pancreatic adenocarcinoma expressing guanylyl cyclase. Investigational New Drugs, 35(5), 634-641.

Al-Musawi, S., Albukhaty, S., Al-Karagoly, H., \& Almalki, F. (2020). Design and synthesis of multi-functional fsuperparamagnetic core-gold shell coated with chitosan and folate nanoparticles for targeted antitumor therapy. Nanomaterials, 11(1), 32 .

Alonso-Álvarez, S., Pardal, E., Sánchez-Nieto, D., Navarro, M., Caballero, M. D. Mateos, M. V., \& Martín, A. (2017). Plitidepsin: Design, development, and potential place in therapy. Drug Design Development and Therapy, 11, 253-264.

Alves, A. J. S., Pereira, J. A., Dethoup, T., Cravo, S., Mistry, S., Silva, A. M. S., Pinto, M. M. M., \& Kijjoa, A. A. (2019). New meroterpene, a new benzofuran derivative and other constituents from cultures of the marine sponge-associated fungus Acremonium persicinum KUFA 1007 and their anticholinesterase activities. Marine Drugs, 17(6), 379.

Amaya, M. L., Jimeno, A., \& Kamdar, M. (2020). Polatuzumab vedotin to treat relapsed or refractory diffuse large B-cell lymphoma, in combination with bendamustine plus rituximab. Drugs Today, 56(4), 287-294.

Andel, L. V., Rosing, H., Schellens, J. H. M., \& Beijnen, J. H. (2018). Review of chromatographic bioanalytical assays for the quantitative determination of marine-derived drugs for cancer treatment. Marine Drugs, 16(7), 246.

Anderson, B. L., Teyton, L., Bendelac, A., \& Savage, P. B. (2013). Stimulation of natural killer T cells by glycolipids. Molecules, 18(12), 15662-15688.

Arumugam, V., Venkatesan, M., Ramachandran, S., \& Sundaresan, U. (2018). Bioactive peptides from marine ascidians and future drug development - a review. International Journal of Peptide Research and Therapeutics, 24(1), 13-18.

Autio, K. A., Boni, V., Humphrey, R. W., \& Naing, A. (2020). Probody therapeutics: An emerging class of therapies designed to enhance on-target effects with reduced off-tumor toxicity for use in immuno-oncology. Clinical Cancer Research, 26(5), 984-989.

Avila, C., \& Angulo-Preckler, C. (2020). Bioactive compounds from marine heterobranchs. Marine Drugs, 18(12), 657.
Babu, A., \& Ramesh, R. (2017). Multifaceted applications of chitosan in cancer drug delivery and therapy. Marine Drugs, 15(4), 96.

Balasubramaniam, V., Gunasegavan, R. D.-N., Mustar, S. S., Lee, J. C., \& Noh, M. F. M. (2021). Isolation of industrial important bioactive compounds from microalgae. Molecules, 26(4), 943.

Barok, M., Puhka, M., Yazdi, N., \& Joensuu, H. (2021). Extracellular vesicles as modifiers of antibody drug conjugate efficacy. Journal Extracell Vesicles, 10(4), e12070.

Barreca, M., Spanò, V., Montalbano, A., Cueto, M., Marrero, A. R. D., Deniz, I., Erdoğan, A., Bilela, L. L., Moulin, C., Taffin-de-Givenchy, E., Spriano, F., Perale, G., Mehiri, M., Rotter, A., Thomas, O. P., Barraja, P., Gaudêncio, S. P., \& Bertoni, F. (2020). Marine anticancer agents: An overview with a particular focus on their chemical classes. Marine Drugs, 18(12), 619.

Belgiovine, C., Bello, E., Liguori, M., Craparotta, I., Mannarino, L., Paracchini, L., Beltrame, L., Marchini, S., Galmarini, C. M., Mantovani, A., Frapolli, R., Allavena, P., \& D'Incalci, M. (2017). Lurbinectedin reduces tumour-associated macrophages and the inflammatory tumour microenvironment in preclinical models. British Joumal of Cancer, 117(5), 628-638.

Bibi, F., Faheem, M., Azhar, E. I., Yasir, M., Alvi, S. A., Kamal, M. A., Ullah, I., \& Naseer, M. I. (2017). Bacteria from marine sponges: A source of new drugs. Current Drug Metabolism, 18(1), 11-15.

Biteghe, N. F. A., Mungra, N., Toung, C. N. E., De La Croix, N. J., EngohangNdong, J., Vignaux, G., Padayachee, E., Naran, K., \& Barth, S. (2020). Advances in epidermal growth factor receptor specific immunotherapy: Lessons to be learned from armed antibodies. Oncotarget, 11(38), 3531-3557.

Blackall, L. L., Wilson, B., \& Van Oppen, M. J. H. (2015). Coral - the world's most diverse symbiotic ecosystem. Molecular Ecology, 24(21), 5330-5347.

Blay, J. Y., Blay, J. Y., Pápai, Z., Tolcher, A. W., Italiano, A., Cupissol, D., LópezPousa, A., Chawla, S. P., Bompas, E., Babovic, N., Penel, N., Isambert, N., Staddon, A. P., Saâda-Bouzid, E., Santoro, A., Franke, F. A., Cohen, P., LeGuennec, S., \& Demetri, G. (2015). Ombrabulin plus cisplatin versus placebo plus cisplatin in patients with advanced soft-tissue sarcomas after failure of anthracycline and ifosfamide chemotherapy: A randomised, double-blind, placebocontrolled, phase 3 trial. The Lancet Oncology, 16(5), 531-540.

Boekhoven, J., Zha, R. H., Tantakitti, F., Zhuang, E., Zandi, R., Newcomb, C. J., \& Stupp, S. I. (2015). Alginate-peptide amphiphile core-shell microparticles as a targeted drug delivery system. RSC Advances, 5(12), 8753-8756.

Boni, V., Meisel, J. L., Tkaczuk, K. H., Wang, Z., Alemany, C., Sterrenberg, D., Wang, Y., Wang, Z., \& Han, H. S. (2019). 196TiPSGNLVA-002: Single arm, open label, phase $\mathrm{Ib} / \mathrm{II}$ study of ladiratuzumab vedotin (LV) in combination with pembrolizumab for first-line treatment of patients with unresectable locally-advanced or metastatic triple-negative breast cancer. Annals of Oncology, 30(3), 63.

Boni, V., Sharma, M. R., \& Patnaik, A. (2020). The resurgence of antibody drug conjugates in cancer therapeutics: Novel targets and payloads. American Society of Clinical Oncology Educational Book, 40, 1-17.

Bourillon, L., Bourgier, C., Gaborit, N., Garambois, V., Llès, E., Zampieri, A., Ogier, C., Jarlier, M., Radosevic-Robin, N., Orsetti, B., Delpech, H., Theillet, C., Colombo, P. E., Azria, D., Pèlegrin, A., Larbouret, C., \& Chardès, T. (2019). An auristatin-based antibody-drug conjugate targeting HER3 enhances the radiation response in pancreatic cancer. International Journal Cancer, 145(7), 1838-1851.

Calcabrini, C., Catanzaro, E., Bishayee, A., Turrini, E., \& Fimognari, C. (2017). Marine sponge natural products with anticancer potential: An updated review. Marine Drugs, $15(10), 310$.

Calvo, E., Moreno, V., Flynn, M., Holgado, E., Olmedo, M. E., Lopez Criado, M. P. Kahatt, C., Lopez-Vilariño, J. A., Siguero, M., Fernandez-Teruel, C., CullellYoung, M., Matos-Pita, A. S., \& Forster, M. (2017). Antitumor activity of lurbinectedin (PM01183) and doxorubicin in relapsed small-cell lung cancer: Results from a phase I study. Annals of Oncology, 28(10), 2559-2566.

Carroll, A. R., Copp, B. R., Davis, R. A., Keyzers, R. A., \& Prinsep, M. R. (2020). Marine natural products. Natural Product Reports, 37, 175-223.

Chakraborty, K., \& Joy, M. (2020). High-value compounds from the molluscs of marine and estuarine ecosystems as prospective functional food ingredients: An overview. Food Research International, 137, 109637.

Chen, R., Herrera, A. F., Hou, J., Chen, L., Wu, J., Guo, Y., Synold, T. W., Ngo, V. N., Puverel, S., Mei, M., Popplewell, L., Yi, S., Song, J. Y., Tao, S., Wu, X., Chan, W. C., Forman, S. J., Kwak, L. W., Rosen, S. T., \& Newman, E. M. (2020). Inhibition of MDR1 overcomes resistance to brentuximab vedotin in Hodgkin lymphoma. Clinical Cancer Research, 26(5), 1034-1044.

Cheng, M.-M., Tang, X.-T., Sun, Y.-T., Song, D.-Y., Cheng, Y.-J., Liu, H., Li, P.-L., \& Li, G.-Q. (2020). Biological and chemical diversity of marine sponge-derived microorganisms over the last two decades from 1998 to 2017. Molecules, 25(4), 853.

Cheng, X., Li, J., Tanaka, K., Majumder, U., Milinichik, A. Z., Verdi, A. C., Maddage, C. J., Rybinski, K. A., Fernando, S., Fernando, D., Kuc, M., Funuchi, K., Fang, F., Uenaka, T., Grasso, L., \& Albone, E. F. (2018). MORAb-202, an antibody-drug conjugate utilizing humanized anti-human FR $\alpha$ farletuzumab and the microtubule-targeting agent eribulin, has potent antitumor activity. Molecular Cancer Therapeutics, 17(12), 2665-2675. 
Cheung, R. C. F., Ng, T. B., \& Wong, J. H. (2015). Marine peptides: Bioactivities and applications. Marine Drugs, 13(7), 4006-4043.

Choi, M. Y., Widhopf, G. F., Ghia, E. M., Kidwell, R. L., Hasan, M. K., Yu, J., Rassenti, L. Z., Chen, L., Chen, Y., Pittman, E., Pu, M., Messer, K., Prussak, C. P., Castro, J. E., Jamieson, C., \& Kipps, T. J. (2018). Phase I trial: Cirmtuzumab inhibits ROR1 signaling and stemness signatures in patients with chronic lymphocytic leukemia. Cell Stem Cell, 22(6), 951-959.

Choi, M. Y., Widhopf, G. F., Wu, C. C. N., Cui, B., Lao, F., Sadarangani, A., Cavagnaro, J., Prussak, C., Carson, D. A., Jamieson, C., \& Kipps, T. J. (2015). Preclinical specificity and safety of UC-961, a first-in-class monoclonal antibody targeting ROR1. Clinical Lymphoma, Myeloma and Leukemia, 15, 167-169.

Choi, Y., \& Diefenbach, C. S. (2020). Polatuzumab vedotin: A new target for B cell malignancies. Current Hematologic Malignancy Reports, 15, 125-129.

Ciavatta, M. L., Lefranc, F., Carbon, M., Mollo, E., Gavagnin, M., Betancourt, T. Dasari, R., Kornienko, A., \& Kiss, R. (2017). Marine mollusk-derived agents with antiproliferative activity as promising anticancer agents to overcome chemotherapy resistance. Medicinal Research Reviews, 37(4), 702-801.

Ciavatta, M. L., Lefranc, F., Vieira, L. M., Kiss, R., Carbone, M., Van Otterlo, W. A. L., Lopanik, N. B., \& Waeschenbach, A. (2020). The phylum Bryozoa: From biology to biomedical potential. Marine Drugs, 18(4), 200.

Cooper, T. M., Absalon, M. J., Alonzo, T. A., Gerbing, R. B., Leger, K. J., Hirsch, B. A., Pollard, J., Razzouk, B. I., Aplenc, R., \& Kolb, E. A. (2020). Phase I/II study of CPX-351 followed by fludarabine, cytarabine, and granulocyte-colony stimulating factor for children with relapsed acute myeloid leukemia: A report from the children's oncology group. Joumal of Clinical Oncology, 38(19), 2170-2177.

Cragg, G. M., Kingston, D. G. I., \& Newman, D. J. (2012). The dolastatins: Novel antitumor agents from Dolabella auricularia. Anticancer Agents from Natural Products. Boca Raton, CRC Press.

De Bono, J. S., Concin, N., Hong, D. S., Thistlethwaite, F. C., Machiels, J.-P., Arkenau, H. T., Plummer, R., Jones, R. H., Nielsen, D., Windfeld, K., Ghatta, S., Slomovitz, B. M., Spicer, J. F., Yachnin, J., Ang, J. E., Mau-Sørensen, P. M., Forster, M. D., Collins, D., Dean, E., Rangwala, R. A., \& Lassen, U. L. (2019). Tisotumab vedotin in patients with advanced or metastatic solid tumours (InnovaTV 201): A first-in-human, multicentre, phase 1-2 trial. Lancet Oncology, 20(3), 383-393.

De Martel, C., Georges, D., Bray, F., Ferlay, J., \& Clifford, G. M. (2020). Global burden of cancer attributable to infections in 2018: A worldwide incidence analysis. Lancet Global Health, 8(2), 180-190.

Demay, J., Halary, S., Knittel-Obrecht, A., Villa, P., Duval, C., Hamlaoui, S., Roussel, T., Yéprémian, C., Reinhardt, A., Bernard, C., \& Marie, B. (2021). Anti-inflammatory, antioxidant, and wound-healing properties of cyanobacteria from thermal mud of Balaruc-Les-Bains, France: A multi-approach study. Biomolecules, 11(1), 28

Deshmukh, S. K., Prakash, V., \& Ranjan, N. (2018). Marine fungi: A source of potential anticancer compounds. Frontiers in Microbiology, 8, 2536.

Diaa, T. A. Y., Almagthali, H., Shaala, L. A., \& Schmidt, E. W. (2020). Secondary metabolites of the genus Didemmum: A comprehensive review of chemical diversity and pharmacological properties. Marine Drugs, 18(6), 307.

Dou, D., \& Dong, B. (2019). Origins and bioactivities of natural compounds derived from marine ascidians and their symbionts. Marine Drugs, 17(12), 670.

Dunsmore, K. P., Winter, S. S., Devidas, M., Wood, B. L., Esiashvili, N., Chen, Z., Eisenberg, N., Briegel, N., Hayashi, R. J., Gastier-Foster, J. M., Carroll, A. J., Heerema, N. A., Asselin, B. L., Rabin, K. R., Zweidler-Mckay, P. A., Raetz, E. A., Loh, M. L., Schultz, K. R., Winick, N. J., Carroll, W. L., \& Hunger, S. P. (2020). Children's oncology group AALL0434: A phase III randomized clinical trial testing nelarabine in newly diagnosed T-cell acute lymphoblastic leukemia. Journal of Clinical Oncology, 38(28), 3282-3293.

Dyshlovoy, S. A., \& Honecker, F. (2015). Marine compounds and cancer: Where do we stand? Marine Drugs, 13(9), 5657-5665.

Dyshlovoy, S. A., \& Honecker, F. (2020). Marine compounds and cancer: Updates. Marine Drugs, 18(12), 643

Eastman, A. (2017). Improving anticancer drug development begins with cell culture: Misinformation perpetrated by the misuse of cytotoxicity assays. Oncotarget, $8(5), 8854-8866$

El Bairi, K., Amrani, M., Kandhro, A. H., Kandhro, A. H. \& Afqir, S. (2017). Prediction of therapy response in ovarian cancer: Where are we now? Critical Reviews in Clinical Laboratory Sciences, 54(4), 233-266.

El-Hossary, E. M., Abdel-Halim, M., Ibrahim, E. S., Pimentel-Elardo, S. M., Nodwell, J. R., Handoussa, H., Abdelwahab, M., Holzgrabe, U., \& Abdelmohsen, U. R. (2020). Natural products repertoire of the Red Sea. Marine Drugs, 18(9), 457.

El-Kashef, D. H., Youssef, F. S., Reimche, I., Teusch, N., Müller, W. E. G., Lin, W., Frank, M., Liu, Z., \& Proksch, P. (2021). Polyketides from the marine-derived fungus Aspergillus falconensis: In silico and in vitro cytotoxicity studies. Bioorganic and Medicinal Chemistry, 29, 115883

Elliot, A., Myllymäki, H., \& Feng, Y. (2020). Inflammatory responses during tumour initiation: From zebrafish transgenic models of cancer to evidence from mouse and man. Cells, 9(4), 1018.
Ercolano, G., De Cicco, P., \& Ianaro, A. (2019). New drugs from the sea: Proapoptotic activity of sponges and algae derived compounds. Marine Drugs, 17(1), 31 .

Esmaeelian, B., Benkendorff, K., Leu, R. K. L., \& Abbott, C. A. (2018). Simultaneous assessment of the efficacy and toxicity of marine mollusc-derived brominated indoles in an in vivo model for early stage colon cancer. Integrative Cancer Therapies, 17(2), 248-262.

Evidente, A., Kornienko, A. Cimmino, A., Andolfi, A., Lefranc, F., Mathieu, V., \& Kiss, R. (2014). Fungal metabolites with anticancer activity. Natural Product Reports, 31, 617-627.

Fadia, S. Y., \& Singab, A. N. B. (2021). An updated review on the secondary metabolites and biological activities of Aspergillus ruber and Aspergillus flavus and exploring the cytotoxic potential of their isolated compounds using virtual screening. Evid Based Complementary and Alternative Medicine, 2021, 8860784.

Fang, W.-Y., Dahiya, R., Qin, H.-L., Mourya, R., \& Maharaj, S. (2016). Natural proline-rich cyclopolypeptides from marine organisms: Chemistry, synthetic methodologies and biological status. Marine Drugs, 14(11), 194.

Ferlay, J., Colombet, M., Soerjomataram, I., Mathers, C., Parkin, D. M., Piñeros, M., Znaor, A., \& Bray, F. (2019). Estimating the global cancer incidence and mortality in 2018: GLOBOCAN sources and methods. International Joumal of Cancer, 144(8), 1941-1953.

Fidler, M. M., Bray, F., \& Soerjomataram, I. (2018). The global cancer burden and human development: A review. Scandinavian Joumal of Public Health, 46(1), $27-36$.

Fitton, J. H., Park, A. Y., Karpiniec, S. S., \& Stringer, D. N. (2020). Fucoidan and lung function: Value in viral infection. Marine Drugs, 19(1), 4.

Fitton, J. H., Stringer, D. N., Park, A. Y., \& Karpiniec, S. S. (2019). Therapies from fucoidan: New developments. Marine Drugs, 17(10), 571.

Forero-Torres, A., Kolibaba, K. S., Lamy, T., Jones, S., Biostat, M. S., Lee, C., \& Sharman, J. (2016). Polatuzumab vedotin combined with obinutuzumab, cyclophosphamide, doxorubicin, and prednisone (G-CHP) for patients with previously untreated diffuse large B-cell lymphoma (DLBCL): Preliminary results of a phase Ib/II dose-escalation study. Blood, 128(22), 1856.

Francisco, J. A., Cerveny, C. G., \& Meyer, D. L. (2003). cAC10-vcMMAE, an antiCD30-monomethyl auristatin E conjugate with potent and selective antitumor activity. Blood, 102(4), 1458-1465.

Frankel, B. M., Cachia, D., Patel, S. J., \& Das, A. (2020). Targeting subventricular zone progenitor cells with intraventricular liposomal encapsulated cytarabine in patients with secondary glioblastoma: A report of two cases. SN Comprehensive Clinical Medicine, 2(6), 836-843.

Frau J., Flores-Holguín, N., \& Glossman-Mitnik, D. (2019). Chemical reactivity theory and empirical bioactivity scores as computational peptidology alternative tools for the Study of two anticancer peptides of marine origin. Molecules, 24(6), 1115.

Fuji, N., Ueda, Y., Fujiwara, H., Toh, T., Yoshimura, T., \& Yamagishi, H. (2000). Antitumor effect of alpha-galactosylceramide (KRN7000) on spontaneous hepatic metastases requires endogenous interleukin 12 in the liver. Clinical Cancer Research, 6(8), 3380-3387.

Gagliardi, A., Giuliano, E., Venkateswararao, E., Fresta, M., Bulotta, S., Awasthi, V., \& Cosco, D. (2021). Biodegradable polymeric nanoparticles for drug delivery to solid tumors. Frontiers in Pharmacology, 12, 601626.

Galasso, C., Gentile, A., Orefice, I., Ianora, A., Bruno, A., Noonan, D. M., Sansone, C., Albini, A., \& Brunet, C. (2019). Microalgal derivatives as potential nutraceutical and food supplements for human health: A focus on cancer prevention and interception. Nutrients, 11(6), 1226.

Gao, J., \& Hamann, M. T. (2011). Chemistry and biology of kahalalides. Chemical Reviews, 111(5), 3208-3235.

Gavriilidou, A., Mackenzie, T. A. M., Sánchez, P., Tormo, J. R., Ingham, C., Smidt, H., \& Sipkema, D. (2021). Bioactivity screening and gene-trait matching across marine sponge-associated bacteria. Marine Drugs, 19(2), 75.

Gentile, D., Patamia, V., Scala, A., Sciortino, M. T., Piperno, A., \& Rescifina, A. (2020). Putative inhibitors of SARS-CoV-2 main protease from a library of marine natural products: A virtual screening and molecular modeling study. Marine Drugs, 2021(19), 64

George, A., Shah, P. A., \& Shrivastav, P. S. (2019). Natural biodegradable polymers based nano-formulations for drug delivery: A review. International Journal of Pharmaceutics, 561, 244-264

Gerber, D. E., Infante, J. R., Gordon, M. S., Goldberg, S. B., Martín, M., Felip, E., Martinez, G. M, Schiller, J. H., Spigel, D. R., Cordova, J., Westcott, V., Wang, Y., Shames, D. S., Choi, Y., Kahn, R., Dere, R. C., Samineni, D., Xu, J., Lin, K., Wood, K., Royer-Joo, S., Lemahieu, V., Schuth, E., Vaze, A., Maslyar, D., Humke, E. W., \& Burris, H. A. (2020). 3rd phase Ia study of anti-NaPi2b antibody-drug conjugate lifastuzumab vedotin DNIB0600A in patients with nonsmall cell lung cancer and platinum-resistant ovarian cancer. Clinical Cancer Research, 26(2), 364-372. 
Gerwick, W. H., \& Moore, B. S. (2012). Lessons from the past and charting the future of marine natural products drug discovery and chemical biology. Journal of Chemical Biology, 19(1), 85-98.

Gnanambal, K. M., \& Lakshmipathy, S. V. (2016). Dictyoceratidan poisons: Defined mark on microtubule-tubulin dynamics. Life Sciences, 148, 229-240.

Gogineni, V. M., \& Hamann, M. T. (2018). Marine natural product peptides with therapeutic potential: Chemistry, biosynthesis, and pharmacology. Biochimica et Biophysica Acta, 1862(1), 81-196.

Golan, T., Khvalevsky, E. Z., Hubert, A., Gabai, R. M., Hen, N., Segal, A., Domb, A., Harari, G., David, E. B., Raskin, S., Goldes, Y., Goldin, E., Eliakim, R., Lahav, M., Kopleman, Y., Dancour, A., Shemi, A., \& Galun, E. (2015). RNAi therapy targeting KRAS in combination with chemotherapy for locally advanced pancreatic cancer patients. Oncotarget, 6(27), 24560-24570.

Gomes, N. G. M., Dasari, R., Chandra, S., Kiss, R., \& Kornienko, A. (2016). Marine invertebrate metabolites with anticancer activities: Solutions to the "Supply problem". Marine Drugs, 14(5), 98.

Gray, B. P., Song, X., Hsu, D. S., Kratschmer, C., Levy, M., Barry, A. P., \& Sullenger, B. A. (2020). An aptamer for broad cancer targeting and therapy. Cancers, 12(11), 3217.

Graziani, E. I., Sung, M., Ma, D., Narayanan, B., Marquette, K., Puthenveetil, S., Tumey, L. N., Bikker, J., Casavant, J., Bennett, E. M., Charati, M. B., Golas, J., Hosselet, C., Rohde, C. M., Hu, G., Guffroy, M., Falahatpisheh, H., Finkelstein, M., Clark, T., Barletta, F., Tchistiakova, L., Lucas, J., Rosfjord, E., Loganzo, F., O’Donnell, C. J., Gerber, H.-P., \& Sapra, P. (2020). PF-06804103, a site-specific anti-HER2 antibody-drug conjugate for the treatment of HER2-expressing breast, gastric, and lung cancers. Molecular Cancer Therapeutics, 19(10), 2068 2078.

Greene, M. K., Chen, T., Robinson, E., Straubinger, N. L., Minx, C., Chan, D. K. W., Wang, J., Burrows, J. F., Van Schaeybroeck, S., Baker, J. R., Caddick, S., Longley, D. B., Mager, D. E., Straubinger, R. M., Chudasama, V. C., \& Scott, C. J. (2020). Controlled coupling of an ultrapotent auristatin warhead to cetuximab yields a next-generation antibody-drug conjugate for EGFR-targeted therapy of KRAS mutant pancreatic cancer. British Journal of Cancer, 123(10), 15021512.

Hamilton, E. P., Barve, M. B., Bardia, A., Beeram, M., Bendell, J. B., Mosher, R., Hailman, E., Bergstrom, D. A., Burris, H. A., \& Soliman, H. H. (2018). Phase 1 dose escalation of XMT-1522, a novel HER2-targeting antibody-drug conjugate (ADC), in patients (pts) with HER2-expressing breast, lung and gastric tumors. Journal of Clinical Oncology, 36(15), 2546-2546.

Hanna, K. S. (2020). Clinical overview of enfortumab vedotin in the management of locally advanced or metastatic urothelial carcinoma. Drugs, 80(1), 1-7.

Hartrampf, N., Seki, T., Baumann, A., Watson, P., Vepřek, N. A., Hetzler, B. E., Hoffmann-Röder, A., Tsuji, M., \& Trauner, D. (2020). Optical control of cytokine production using photoswitchable galactosylceramides. Chemistry, 26(20), $4476-4479$.

Hasanov, M., Rioth, M. J., Kendra, K., Hemandez-Aya, L., Joseph, R. W., Williamson, S., Chandra, S., Shirai, K., Tumer, C. D., Lewis, K., Crowley, E., Moscow, J., Carter, B., \& Patel, S. (2020). A phase II study of glembatumumab vedotin for metastatic uveal melanoma. Cancers, 12(8), 2270.

He, C., Hua, X., Sun, S., Li, S., Wang, J., \& Huang, X. (2021). Integrated bioinformatic analysis of SARS-CoV-2 infection related genes ACE2, BSG and TMPRSS2 in aerodigestive cancers. Inflammation Researchs, 10(14), 791-802.

Hingorani, D. V., Doan, M. K., Camargo, M. F., Aguilera, J., Song, S. M., Pizzo, D., Scanderbeg, D. J., Cohen, E. E. W., Lowy, A. M., Adams, S. R., \& Advani, S. J. (2020). Precision chemoradiotherapy for HER2 tumors using antibody conjugates of an auristatin derivative with reduced cell permeability. Molecular Cancer Therapeutics, 19(1), 157-167.

Hingorani, P., Roth, M. E., Wang, Y., Zhang, W., Gill, J. B., Harrison, D. J., Teicher, B., Erickson, S., Gatto, G., Smith, M. A., Kolb, E. A., \& Gorlick, R. (2021). ABBV-085, antibody-drug conjugate targeting LRRC15, is effective in osteosarcoma: A report by the pediatric preclinical testing consortium. Molecular Cancer Therapeutics, 20(3), 535-540.

Hong, D. S., Nicole Concin, N., Vergote, I., De Bono, J., Slomovitz, B. M., Drew, Y., Arkenau, H.-T., Machiels, J.-P., Spicer, J. F., Jones, R., Forster, M. D., Cornez, N., Gennigens, C., Johnson, M., Thistlethwaite, F. C., Rangwala, R. A. Ghatta, S. G., Windfeld, K., Harris, J. R., Lassen, U. N., \& Coleman, R. L. (2020). Tisotumab vedotin in previously treated recurrent or metastatic cervical cancer. Clinical Cancer Research, 26(6), 1220-1228.

Hou, X.-M., Hai, Y., Gu, Y.-C., Wang, C.-Y., \& Shao, C.-L. (2019). Chemical and bioactive marine natural products of coral-derived microorganisms (20152017). Current Medicinal Chemistry, 26(38), 6930-6941.

Hsu, H.-Y., \& Hwang, P.-A. (2019). Clinical applications of fucoidan in translational medicine for adjuvant cancer therapy. Clinical and Translational Medicine, $8(1), 15$.

Islam, M. M., Shahruzzaman, M., Biswas, S., Sakib, N. M., \& Rashid, T. U. (2020). Chitosan based bioactive materials in tissue engineering applications - a review. Bioactive Materials, 5(1), 164-183.
Islam, M. S., Haque, P., Rashid, T. U., Khan, M. N., Mallik, A. M., Khan, M. N. I., \& Rahman, M. M. (2017). Core-shell drug carrier from folate conjugated chitosan obtained from prawn shell for targeted doxorubicin delivery. Joumal of Materials Science: Materials in Medicine, 28(4), 55.

Jensen, P. R., Moore, B. S., \& Fenical, W. (2015). The marine actinomycete genus Salinispora: A model organism for secondary metabolite discovery. Natural Product Reports, 32(5), 738-751.

Jimenez, P. C., Wilke, D. V., Branco, P. C., Bauermeister, A., Rezende-Teixeira, P., Gaudêncio, S. P., \& Costa, L. V. (2020). Enriching cancer phamacology with drugs of marine origin. British Journal of Pharmacology, 177(1), 3-27.

Johansson, M. P., Maaheimo, H., \& Ekhol, F. S. (2017). New insight on the structural features of the cytotoxic auristatins MMAE and MMAF revealed by combined NMR spectroscopy and quantum chemical modelling. Scientific Reports, 7, 15920 .

Jones, E. B. G., Suetrong, S., \& Sakayaroj, J. (2015). Classification of marine Ascomycota, Basidiomycota, Blastocladiomycota and Chytridiomycota. Fungal Diversity, 73, 1-72.

Jones, L., McCalmont, H., Evans, K., Mayoh, C., Kurmasheva, R. T., Billups, C. A., Houghton, P. J., Smith, M. A., \& Lock, R. B. (2019). Preclinical activity of the antibody-drug conjugate denintuzumab mafodotin (SGN-CD19A) against pediatric acute lymphoblastic leukemia xenografts. Pediatric Blood and Cancer, 66(8), 27765

Kadia, T. M., \& Gandhi, V. (2017). Nelarabine in the treatment of pediatric and adult patients with T-cell acute lymphoblastic leukemia and lymphoma. Expert Review of Hematology, 10(1), 1-8.

Kalhotra, P., Veera, C. S. R. Chittepu, V. C. S. R., Osorio-Revilla, G., \& GallardoVelazquez, T. (2021). Field-template, QSAR, ensemble molecular docking, and 3D-RISM solvation studies expose potential of FDA-approved marine drugs as SARS-CoVID-2 main protease inhibitors. Molecules, 26(4), 936

Kang, H. K., Choi, M. C., \& Seo, C. H. (2018). Therapeutic properties and biological benefits of marine-derived anticancer peptides. International Joumal of Molecular Sciences, 19(3), 919.

Kaštelan, S., Antunica, A. G., Oresković, L. B., Pelčić, G., Kasun, E., \& Hat, K. (2020). Immunotherapy for uveal melanoma - current knowledge and perspectives. Current Medicinal Chemistry, 27(8), 1350-1366.

Katoh, M. (2017). Canonical and non-canonical WNT signaling in cancer stem cells and their niches: Cellular heterogeneity, omics reprogramming, targeted therapy and tumor plasticity (review). International Joumal of Oncology, 51(5), 13571369.

Kauffmann-Guerrero, D., \& Huber, R. M. (2020). Orphan drugs in development for the treatment of small-cell lung cancer: Emerging data on lurbinectedin. Lung Cancer, 11, 27-31.

Kellogg, C. A. (2019). Microbiomes of stony and soft deep-sea corals share rare core bacteria. Microbiome, 7,90.

Khan, P., Siddiqui, J. A., Lakshmanan, I., Ganti, A. K., Salgia, R., Jain, M., Batra, S. K., \& Nasser, M. W. (2021). RNA-based therapies: A cog in the wheel of lung cancer defense. Molecular Cancer, 20, 54.

Khongorzul, P., Ling, C. J., Khan, F. U., Ihsan, A. U. I., \& Zhang, J. (2020). Antibody-drug conjugates: A comprehensive review. Molecular Cancer Research, 18(1), 3-19.

Kim, Y. C., \& Guan, K. L. (2015). mTOR: A pharmacologic target for autophagy regulation. Joumal of Clinical Investigation, 125(1), 25-32.

Knobloch, S., Jóhannsson, R., \& Marteinsson, V. (2019). Co-cultivation of the marine sponge Halichondria panicea and its associated microorganisms. Scientific Reports, 9(1), 10403.

Knobloch, S., Jóhannsson, R., \& Marteinsson, V. (2020). Genome analysis of sponge symbiont 'Candidatus Halichondribacter symbioticus' shows genomic adaptation to a host-dependent lifestyle. Environmental Microbiology, 22(1), 483-498.

Kobayashi, H., Iwata, S., Wakamatsu, T., Hayakawa, K., Yonemoto, T., Wasa, J., Oka, H., Ueda, T., \& Tanaka, S. (2020). Efficacy and safety of trabectedin for patients with unresectable and relapsed soft-tissue sarcoma in Japan: A japanese musculoskeletal oncology group study. Cancer, 126(6), 1253-1263.

Kollmannsberger, C., Choueiri, T. K., Heng, D. Y. C., George, S., Jie, F., Croitoru, R., Poondru, S., \& Thompson, J. A. (2021). A randomized phase II study of AGS $16 \mathrm{C} 3 \mathrm{~F}$ versus axitinib in previously treated patients with metastatic renal cell carcinoma. Oncologist, 26(3), 182-361.

Koopman, L. A., Terp, M. G., Zom, G. Z., Janmaat, M. L., Jacobsen, K., Van den Heuvel, E. G., Brandhorst, M., Forssmann, U., De Bree, F., Pencheva, N., Lingnau, A., Zipeto, M. A., Parren, P. W. H. I., Breij, E. C. W., \& Ditzel, H. J. (2019). Enapotamab vedotin, an AXL-specific antibody-drug conjugate, shows preclinical antitumor activity in non-small cell lung cancer. Insight. Journal of Clinical Investigation Insight, 4(21), 128199.

Lammers, R. J., Witjes, W. P., Janzing-Pastors, M. H., Christien, T. M. C., \& Witjes, J. A. (2012). Intracutaneous and intravesical immunotherapy with keyhole limpet hemocyanin compared with intravesical mitomycin in patients with nonmuscle-invasive bladder cancer: Results from a prospective randomized phase III trial. Joumal of Clinical Oncology, 30, 2273-2279. 
Le Joncour, V., Martins, A., Puhka, M., Isola, J., Salmikangas, M., Laakkonen, P., Joensuu, H., \& Barok, M. (2019). A novel anti-HER2 antibody-drug conjugate XMT-1522 for HER2-positive breast and gastric cancers resistant to trastuzumab emtansine. Molecular Cancer Therapeutics, 18(10), 1721-1730.

Leea, Y., Phatb, C., \& Hong, S.-C. (2017). Structural diversity of marine cyclic peptides and their molecular mechanisms for anticancer, antibacterial, antifungal, and other clinical applications. Peptides, 95, 94-105.

Li, L., Xu, M.-Z., Wang, L., Jiang, J., Dong, L.-H., Chen, F., Dong, K., \& Song, H.F. (2020). Conjugating MMAE to a novel anti-HER2 antibody for selective targeted delivery. European Review for Medical and Pharmacological Sciences, 24(24), 12929-12937.

Li, P., Li, L., Wang, S., Liu, Y., Li, Z., \& Xia, S. (2021). Effect of antitumor therapy on cancer patients infected by SARS-CoV-2: A systematic review and metaanalysis. Cancer Medicine, 10(5), 1644-1655.

Liersch, T., Meller, J., Kulle, B., Behr, T. M., Markus, P., Langer, C., Ghadimi, B. M., Wegener, W. A., Kovacs, J. K., Horak, I. D., Becker, H., \& Goldenberg, D. M. (2005). Phase II trial of carcinoembryonic antigen radioimmunotherapy with 131I-labetuzumab after salvage resection of colorectal metastases in the liver: Five-year safety and efficacy results. Joumal of Clinical Oncology, 23(27), 6763-6770.

Lin, Y., Qi, X., Liu, H., Xue, K., Xu, S., \& Tian, Z. (2020). The anti-cancer effects of fucoidan: A review of both in vivo and in vitro investigations. Cancer Cell International, 20, 154 .

Liu, J. F., Moore, K. N., Birrer, M. J., Berlin, S., Matulonis, U. A., Infante, J. R., Wolpin, B., Poon, K. P., Firestein, R., Xu, J., Kahn, R., Wang, Y., Wood, K., Darbonne, W. C., Lackner, M. R., Kelley, S. K., Lu, X., Choi, Y. J., Maslyar, D., Humke, E. W., \& Burris, H. A. (2016). Phase I study of safety and pharmacokinetics of the anti-MUC16 antibody-drug conjugate DMUC5754A in patients with platinum-resistant ovarian cancer or unresectable pancreatic cancer. Annals of Oncology, 27(11), 2124-2130.

Liu, J., Pandya, P., \& Afshar, S. (2021). Therapeutic advances in oncology. International Journal of Molecular, 22(4), 2008.

Liu, Z., Frank, M., Yu, X., Yu, H., Tran-Cong, N. M., Gao, Y., \& Proksch, P. (2020). Secondary metabolites from marine-derived fungi from China. Progress in the Chemistry of Organic Natural Products, 111, 81-153.

López-Iglesias, A.-A., González-Méndez, L., San-Segundo, L., Herrero, A. B., Hernández-García, S., Martín-Sánchez, M., Gutiérrez, N. C., Paíno, T., Avilés, P., Mateos, M.-V., San-Miguel, J. F., Garayoa, M., \& Ocio, E. M. (2017). Synergistic DNA-damaging effect in multiple myeloma with the combination of zalypsis, bortezomib and dexamethasone. Haematologica, 102(1), 168-175.

Losada, A., Berlanga, J. J., Molina-Guijarro, J. M., Jiménez-Ruiz, A., Gago, F., Avilés, P., De Haro, C., \& Martínez-Lea, J. F. (2020). Generation of endoplasmic reticulum stress and inhibition of autophagy by plitidepsin induces proteotoxic apoptosis in cancer cells. Biochemical Pharmacology, 172, 113744

Lowe, K. L., Mackall, C. L., Norry, E., Amado, R., Jakobsen, B. K., \& Binder, G. (2018). Fludarabine and neurotoxicity in engineered T-cell therapy. Gene Therapy, 25(3), 176-191.

Luque-Bolivar, A., Pérez-Mora, E., Villegas, V. E., \& Rondón-Lagos, M. (2020). Resistance and overcoming resistance in breast cancer. Breast Cancer, 12, 211-229.

Ma, H. L., Xu, Y. F., Qi, X. R., Maitani, Y., \& Nagai, T. (2008). Superpara magnetic iron oxide nanoparticles stabilized by alginate: Pharmacokinetics, tissue distribution, and applications in detecting liver cancers. International Joumal of Pharmaceutics, 354, 217-226.

Maderna, A., Doroski, M., Subramanyam, C., Porte, A., Leverett, C. A., Vetelino, B. C., Chen, Z., Risley, H., Parris, K., Pandit, J., Varghese, A. H., Shanker, S., Song, C., Sukuru, S. C. K., Farley, K. A., Wagenaar, M. M., Shapiro, M. J., Musto, S., Lam, M.-H., Loganzo, F., \& O'Donnell, C. J. (2014). Discovery of cytotoxic dolastatin 10 analogues with $\mathrm{N}$-terminal modifications. Journal of Medicinal Chemistry, 57(24), 10527-10543.

Manzano, A., \& Ocaña, A. (2222). Antibody-Brug conjugates: A promising novel therapy for the treatment of ovarian cancer. Cancers, 12(8), 2223.

Marchetti, P., Pavan, B., Simoni, D., Baruchello, R., Rondanin, R., Mischiati, C., Feriotto, G., Ferraro, L., Hsu, L.-C., Lee, R. M., \& Dalpiaz, A. (2016). A novel hybrid drug between two potent anti-tubulin agents as a potential prolonged anticancer approach. European Journal of Pharmaceutical Sciences, 91, 50-63.

Markham, A. (2020). Lurbinectedin: First approval. Drugs, 80(13), 1345-1353.

Martínez Andrade, K. A., Lauritano, C., Romano, G., \& Ianora, A. (2018). Marine microalgae with anti-cancer properties. Marine Drugs, 16(5), 165.

Martínez-Trufero, J., De Sande-González, L. M., Luna, P., Martin-Broto, J., Álvarez, R., Marquina, G., Diaz-Beveridge, R., Poveda, A., Cano, J. M., Cruz-Jurado, J., Pousa, A. L., Salgado, M. A. V., Valverde-Morales, C. M., Sevilla, I., MartínezGarcía, J., Rubio-Casadevall, J., De Juan, A., Carrasco, J. A., Moura, D. S., Gurruchaga-Sotes, I., \& Gutiérrez, A. (2021). A growth modulation index-based GEISTRA score as a new prognostic tool for trabectedin efficacy in patients with advanced soft tissue sarcomas: A spanish group for sarcoma research (GEIS) retrospective study. Cancers, 13(4), 792.
Martins, A., Vieira, H., Gaspar, H., \& Santos, S. (2014). Marketed marine natural products in the pharmaceutical and cosmeceutical industries: Tips for success. Marine Drugs, 12(2), 1066-1101.

Matsunaga, Y., Yamaoka, T., Ohba, M., Miura, S., Masuda, H., Sangai, T., Takimoto, M., Nakamura, S., \& Tsurutani, J. (2021). Novel anti-FOLR1 antibody-drug conjugate MORAb-202 in breast cancer and non-small cell lung cancer cells. Antibodies, 10(1), 6

McCarthy, P. J., Roberts, B. F., Carbonell, A., Roberts, J., Wright, A. E., \& Chakrabarti, D. (2019). Marine microbiome as a source of antimalarials. Tropical Medicine and Infectious Disease, 4(3), 103.

McNemey, O. K., \& Teachey, D. T. (2021). Xenograft models for pediatric cancer therapies. Faculty Reviews, 10, 11.

Mehbub, M. F., Lei, J., Franco, C., Franco, C., \& Zhang, W. (2014). Marine sponge derived natural products between 2001 and 2010: Trends and opportunities for discovery of bioactives. Marine Drugs, 12(8), 4539-4577.

Menck, K., Heinrichs, S., Baden, C., \& Bleckmann, A. (2021). The WNT/ROR pathway in cancer: From signaling to therapeutic intervention. Cells, 10(1), 142.

Meric-Bernstam, F., Calvo, E., Moreno, V., Chung, H. C., Park, Y. H. P., Bang, Y.J., Rosen, L. S., Mita, M. M., Garrido-Laguna, I., Leung, A. C. F., Dube, H., Zhong, W., Chen, X., Dawaher, R., \& Curigliano, G. (2020). A phase I dose escalation study evaluating the safety and tolerability of a novel anti-HER2 antibody-drug conjugate (PF-06804103) in patients with HER2-positive solid tumors. Journal of Clinical Oncology, 38(15), 1039.

Miller, J. H., Field, J. J., Kanakkanthara, A., Owen, J. G., Singh, A. J., \& Northcote, P. T. (2018). Marine invertebrate natural products that target microtubules. Joumal of Natural Products, 81(3), 691-702.

Miwa, S., Yamamoto, N., Hayashi, K., Takeuchi, A., Igarashi, K., \& Tsuchiya, H. (2019). Therapeutic targets for bone and soft-tissue sarcomas. International Journal of Molecular Sciences, 20(1), 170.

Miyata, Y., Matsuo, T., Ohba, K., Mitsunari, K., Mukae, Y., Otsubo, A., Harada, J., Matsuda, T., Kondo, T., \& Sakai, H. (2020). Present status, limitations and future directions of treatment strategies using fucoidan-based therapies in bladder cancer. Cancers, 12(12), 3776.

Moeller, M., Nietzer, S., \& Schupp, P. J. (2019). Neuroactive compounds induce larval settlement in the scleractinian coral Leptastrea purpurea. Scientific Reports, 9(1), 2291.

Moore, K. N., Birrer, M. J., Marsters, J., Wang, Y., Choi, Y., Royer-Joo, S., Lemahieu, V., Armstrong, K., Cordova, J., Samineni, D., Schuth, E., Vaze, A., Maslyar, D., Humke, E. W., Hamilton, E. P., \& Liu, J. F. (2020). Phase 1b study of anti-NaPi2b antibody-drug conjugate lifastuzumab vedotin (DNIB0600A) in patients with platinum-sensitive recurrent ovarian cancer. Gynecologic Oncology, 158(3), 631-639.

Morell, M., Novotná, E., Milan, J., Danielisová, P., Büküm, N., \& Wsól, V. (2021) Selective inhibition of aldo-keto reductase 1C3: A novel mechanism involved in midostaurin and daunorubicin synergism. Archives of Toxicology, 95(1), 67-78.

Morschhauser, F., Flinn, I. W., Advani, R., Sehn, L. H., Diefenbach, C., Kolibaba, K., Press, O. W., Salles, G., Tilly, H., Chen, A. I., Assouline, S., Cheson, B. D., Dreyling, M., Hagenbeek, A., Zinzani, P. L., Jones, S., Cheng, J., Lu, D., Penuel, E., Hirata, J., Wenger, M., Chu, Y. W., \& Sharman, J. (2019). Polatuzumab vedotin or pinatuzumab vedotin plus rituximab in patients with relapsed or refractory non-Hodgkin lymphoma: Final results from a phase 2 randomised study (ROMULUS). Lancet Haematology, 6(5), 254-265.

Mukherjee, A., Waters, A. K., Babic, I., Nurmemmedov, E., Glassy, M. C., Kesari, S., \& Yenugonda, V. M. (2019). Antibody drug conjugates: progress, pitfalls, and promises. Human Antibodies. 27(1), 53-62.

Müller, P., Martin, K., Theurich, S., Schreiner, J., Savic, S., Terszowski, G., Lardinois, D., Heinzelmann-Schwarz, V. A., Schlaak, M., Kvasnicka, H.-M., Spagnoli, G., Dimhofer, S., Speiser, D. E., Von Bergwelt-Baildon, M., \& Zippelius, A. (2014). Microtubule-depolymerizing agents used in antibody-drug conjugates induce antitumor immunity by stimulation of sendritic cells. Cancer Immunology Research, 2(8), 741-55.

Musaimi, O. A., Shaer, D. A., Albericio, F., \& De la Torre, B. G. (2021). 2020 FDA TIDES (peptides and oligonucleotides) harvest. Pharmaceuticals, 14(2), 145.

Negi, B., Kumar, D. S., \& Rawat, D. (2017). Marine peptides as anticancer agents: A remedy to mankind by nature. Current Protein and Peptide Science, 18(9), 885-904.

Nejadmoghaddam, M. R., Minai-Tehrani, A., Ghahremanzadeh, R., Mahmoudi, M., Dinarvand, R., \& Zamani, A.-H. (2019). Antibody-drug conjugates: Possibilities and challenges. Avicenna Journal of Medical Biotechnology, 11(1), 3-23.

Newman, D. J. (2016). Developing natural product drugs: Supply problems and how they have been overcome. Pharmacology and Therapeutics, 162, 1-9.

Newman, D. J. (2019). The "utility" of highly toxic marine-sourced compounds. Marine Drugs, 17(6), 324.

Newman, D. J., \& Cragg, G. M. (2014). Marine-sourced anti-cancer and cancer pain control agents in clinical and late preclinical development. Marine Drugs, 12(1), 255-278. 
Newman, D. J., \& Cragg, G. M. (2017). Current status of marine-derived compounds as warheads in anti-tumor drug candidates. Marine Drugs, 15(4), 1-19.

Novoselova, M. V., German, S. V., Abakumova, T. O., Perevoschikov, S. V., Sergeeva, O. S., Nesterchuk, M. V., Efimov, O. I., Petrov, K. S., Chemysheva, V. S., Zatsepin, T. S., \& Gorin, D. G. (2021). Multifunctional nanostructured drug delivery carriers for cancer therapy: Multimodal imaging and ultrasound-induced drug release. Colloids Surfaces B Biointerfaces, 200, 111576.

Ocio, E. M., Oriol, A., Bladé, J., Teruel, A. I., Martín, J., De la Rubia, J., Gutiérrez, N. C., Díaz-Pavón, J. R., González, S. M., Coronado, C., Fernández-García, E. M., Gómez, M. S., Fernández-Teruel, C., \& Miguel, J. S. (2016). Phase I/II study of weekly PM00104 (zalypsis) in patients with relapsed/refractory multiple myeloma. British Joumal of Haematology, 172(4), 625-628.

Ogaki, M. B., Coelho, L. C., Vieira, R., Neto, A. A., Zani, C. L., Alves, T. M. A., Junior, P. A. S., Murta, S. M. F., Barbosa, E. C., Oliveira, J. G., Ceravolo, I. P., Pereira, P. O., Cota, B. B., Viana, R. O., Alves, V. S., \& Rosa, L. H. (2020). Cultivable fungi present in deep-sea sediments of Antarctica: Taxonomy, diversity, and bioprospecting of bioactive compounds. Extremophiles, 24(2), 227-238.

Orgueira, A. M., Pérez, L. B. P., Torre, A. M. T., Raíndo, A. P. R., López, M. C. L., Arias, J. A. D., Ferro, R. F. F., Rodríguez, B. A. R., Pérez, M. S. G. P., Ferreiro, M. A. F., Vence, N. A. V., Encinas, M. M. P. E., López, J. L. B. L., Martinelli, G., \& Cerchione, C. (2020). FLT3 inhibitors in the treatment of acute myeloid leukemia: Current status and future perspectives. Minerva Medica, 111(5), 427-442.

Ou, J., Liu, K., Jiang, J., Wilson, D. A., Liu, L., Wang, F., Wang, S., Tu, Y., \& Peng, F. (2020). Micro/nanomotors toward biomedical applications: The recent progress in biocompatibility. Review. Nano-Micro Small, 16(27), 1906184.

Palanisamy, S. K., Rajendran, N. M., \& Marino, A. (2017). Natural products diversity of marine ascidians (Tunicates; Ascidiacea) and successful drugs in clinical development. Natural Products and Bioprospecting, 7(1), 1-111.

Park, Y. H., Ahn, H. K., Kim, J.-Y., Ahn, J. S., Im, Y.-H., Kim, S.-H., Lee, S., Chung, H.-S., \& Park, S. J. (2020). First-in-human phase I study of ALT-P7, a HER2-targeting antibody-drug conjugate in patients with HER2-positive advanced breast cancer. Journal of Clinical Oncology, 38(15), 3551.

Patrick, A. O., Pavlick, A. C., Johnson, D. B., Hart, L. L., Infante, J. R., Luke, J. J., Lutzky, J., Rothschild, N. E., Spitler, L. E., Cowey, C. L., Alizadeh, A. R., Salama, A. K., He, Y., Hawthome, T. R., Bagley, R. G., Zhang, J., Turner, C. D. \& Hamid, O. (2019). A phase 2 study of glembatumumab vedotin, an antibodydrug conjugate targeting glycoprotein $\mathrm{NMB}$, in patients with advanced melanoma. The Cancer Journal, 125(7), 1113-1123.

Pereira, D. S., Guevara, C. I., Jin, L., Mbong, N., Verlinsky, A., Hsu, S., Aviña, H Karki, S., Abad, J. D., Yang, P., Moon, S.-J., Malik, F., Choi, M. Y. M., An, Z. Morrison, K., Challita-Eid, P. M., Doñate, F., Joseph, I. B. J., Kipps, T. J., Dick, J. E., \& Stover, D. R. (2015). AGS67E, an anti-CD37 monomethyl auristatin E antibody-drug conjugate as a potential therapeutic for B/T-Cell malignancies and AML: A new role for CD37 in AML. Molecular Cancer Therapeutics, 14(7), 1650-1660.

Pereira, R. B., Evdokimov, N. M., Lefranc, F., Valentão, P., Kornienko, A., Pereira, D. P., Andrade, P. B., \& Gomes, N. G. M. (2019). Marine-derived anticancer agents: Clinical benefits, innovative mechanisms, and new targets. Marine Drugs, 17(6), 329

Perez, E. A., Hillman, D. W., Fishkin, P. A., Krook, J. E., Tan, W. W., Kuriakose, P. A., Alberts, S. R., \& Dakhil, S. R. (2005). Phase II trial of dolastatin-10 in patients with advanced breast cancer. Investigational New Drugs, 23(3), 257-261.

Pérez-Pérez, M. J., Priego, E. M., \& Bueno, O. (2016). Blocking blood flow to solid tumors by destabilizing tubulin: An approach to targeting tumor growth. Journal of Medicinal Chemistry, 59(19), 8685-8711.

Petek, B. J., \& Jones, R. L. (2014). PM00104 (Zalypsis $\left.{ }^{\circledR}\right)$ ): A marine derived alkylating agent. Molecules, 19(8), 12328-12335.

Petrova, G. V., Kaprin, A. D, Grecova, O. P., \& Starinskij, V. V. (2015) Zlokachestvennye novoobrazovanija v Rossii: Obzor statisticheskoj informacii za 1993 2013 gg. [Malignant neoplasms in Russia: Review of statistical information for 1993-2013]. MNIOI im. P. A. Gercena, Moscow (in Russian).

Petrul, H. M., Schatz, C. A., Kopitz, C. C., Adnane, L., McCabe, T. J., Trail, P., Ha, S., Chang, Y. S., Voznesensky, A., Ranges, G., \& Tamburini, P. P. (2012). Therapeutic mechanism and efficacy of the antibody-drug conjugate BAY 79-4620 targeting human carbonic anhydrase 9. Molecular Cancer Therapeutics, 11(2), 340-349.

Petrylak, D. P., Heath, E. I., Sonpavde, G., George, S., Morgans, A. K., Eigl, B. J., Picus, J., Cheng, S. Y., Hotte, S. J., Gartner, E. M., Vincent, M., Chu, R., Anand, B., Morrison, K., Jackson, L., Reyno, L. M., \& Yu, E. (2016). Antitumor activity, safety and pharmacokinetics (PK) of AGS15E (ASG-15ME) in a phase I dose escalation trial in patients (Pts) with metastatic urothelial cancer (mUC). Joumal of Clinical Oncology, 34(15), 4532

Petrylak, D. P., Vogelzang, N. J., Chatta, K., Fleming, M. T., Smith, D. C., Appleman, L. J., Hussain, A., Modiano, M., Singh, P., Tagawa, S. T., Gore, I., McClay, E. F., Mega, A. E., Sartor, A. O., Somer, B., Wadlow, R., Shore, N. D., Olson, W. C., Stambler, N., DiPippo, V. A., \& Israel, R. J. (2020). PSMA ADC monotherapy in patients with progressive metastatic castration-resistant prostate can- cer following abiraterone and/or enzalutamide: Efficacy and safety in open-label single-arm phase 2 study. Prostate, 80(1), 99-108.

Petty, R., Anthoney, A., Metges, J. P., Alsina, M., Gonçalves, A., Brown, J., Montagut, C., Gunzer, K., Laus, G., Dios, J. L. I., Miguel-Lillo, B., Bohan, P., \& Salazar, R. (2016). Phase Ib/II study of elisidepsin in metastatic or advanced gastroesophageal cancer (IMAGE trial). Cancer Chemotherapy and Pharmacology, 77(4), 819-827.

Pimenoff, V. N., Tous, S., Benavente, Y., Alemany, L., Quint, W., Bosch, F. X., Bravo, I. G., \& De Sanjosé, S. (2019). Distinct geographic clustering of oncogenic human papillomaviruses multiple infections in cervical cancers: Results from a worldwide cross-sectional study. International Joumal of Cancer, 144(10), 2478-2488.

Pivkin, M. V., Kuznecova, T. A., \& Sova, V. V. (2006). Morskie griby i ih metabolity [Marine fungi and their metabolites]. Dal'nauka, Vladivostok (in Russian).

Polu, K. R., \& Lowman, H. B. (2014). Probody therapeutics for targeting antibodies to diseased tissue. Expert Opinion on Biological Therapy, 14(8), 1049-1053.

Ponziani, S., Di Vittorio, G., Pitari, G., Cimini, A. C., Ardini, M., Gentile, R., Iacobelli, S., Sala, G., Capone, E., Flavell, D. J., Ippoliti, R., \& Giansanti, F. (2020). Antibody-drug conjugates: The new frontier of chemotherapy. International Journal of Molecular Sciences, 21(15), 5510.

Popov, A. M. (2006). Protivoopuholevaja aktivnost' vtorichnyh metabolitov morskih bespozvonochnyh [Antitumor activity of secondary metabolites of marine invertebrates]. Vestnik DVO RAN, 5, 81-90 (in Russian).

Prota, A. E., Bargsten, K., Diaz, J. F., Marsh, M., Cuevas, C., Liniger, M., Neuhaus, C., Andreu, J., Altmann, K.-H., \& Steinmetz, M. O. (2014). A new tubulin-binding site and pharmacophore for microtubule-destabilizing anticancer drugs. Proceedings of the National Academy of Sciences USA, 111(38), 13817-13821.

Purcell, J. W., Tanlimco, S. G., Hickson, J., Fox, M., Sho, M., Durkin, L., Uziel, T., Powers, R., Foster, K., McGonigal, T., Kumar, S., Samayoa, J., Zhang, D., Palma, J. P., Mishra, S., Hollenbaugh, D., Gish, K., Morgan-Lappe, S. E., His, E. D., \& Chao, D. T. (2018). LRRC15 is a novel mesenchymal protein and stromal target for antibody-drug conjugates. Cancer Research, 78(14), 4059 4072 .

Qamar, H., Hussain, K, Soni, A, Khan, A, Hussain, T, \& Chénais, B. (2021) Cyanobacteria as natural therapeutics and pharmaceutical potential: Role in antitumor activity and as nanovectors. Molecules, 26(1), 247.

Raghuvanshi, R., \& Bharate, S. B. (2020). Preclinical and clinical studies on bryostatins, a class of marine-derived protein kinase $\mathrm{C}$ modulators: A mini-review. Current Topics in Medicinal Chemistry, 20(12), 1124-1135.

Rago, B., Tumey, L. N., Wei, C., Barletta, F., Clark, T., Hansel, S., \& Han, X. (2017). Quantitative conjugated payload measurement using enzymatic release of antibody-drug conjugate with cleavable linker. Bioconjugate Chemistry, 28(2), $620-626$.

Ratnayake, R., Gunasekera, S. P., Ma, J. J., Dang, L. H., Carney, T. J., Paul, V. J., \& Luesch, H. (2020). Dolastatin 15 from a marine cyanobacterium suppresses $\mathrm{HIF}-1 \alpha$ mediated cancer cell viability and vascularization. ChemBioChem, 21(16), 2356-2366.

Ray, A., Okouneva, T., Manna, T., Miller, H. P., Schmid, S., Arthaud, L., Luduena, R., Jordan, M. A., \& Wilson, L. (2007). Mechanism of action of the microtubule-targeted antimitotic depsipeptide tasidotin (formerly ILX651) and its major metabolite tasidotin C-carboxylate. Cancer Research, 67(8), 3767-3776.

Remize, M., Brunel, Y., Silva, J. J., \& Berthon, J.-Y., Filaire, E. (2021). Microalgae $\mathrm{n}-3$ PUFAs production and use in food and feed industries. Marine Drugs, 19(2), 113.

Riccio, G., Ruocco, N., Mutalipassi, M., Costantini, M., Zupo, V., Coppola, D., Pascale, D. D., \& Lauritano, C. (2020). Ten-year research update review: Antiviral activities from aarine organisms. Biomolecules, 10(7), 1007.

Rinnerthaler, G., Gampenrieder, S. P., \& Greil, P. (2019). HER2 directed antibodydrug-conjugates beyond T-DM1 in breast cancer. International Journal of Molecular Sciences, 20(5), 1115.

Roach, T. N. F., Little, M., Arts, M. G. I., Huckeba, J., Haas, H. J., George, E. E. Quinn, R. A., Cobián-Güemes, A. G., Naliboff, D. S., Silveira, C. B., Vermeij, M. J. A., Kelly, L. W., Dorrestein, P. C., \& Rohwer, F. (2020). A multiomic analysis of in situ coral-turf algal interactions. Proceedings of the National Academy of Sciences of the United States of America, 117(24), 13588-13595.

Roberts, T. C., Langer, R., \& Matthew, J. A. (2020). Advances in oligonucleotide drug delivery. Nature Reviews Drug Discovery, 19(10), 673-694.

Rocha-Lima, C. M., Bayraktar, S., Macintyre, J., Raez, L., Flores, A., Ferrell, A., Rubin, E. H., Poplin, E. A., Tan, A. R., Lucarelli, A., \& Zojwalla, N. (2010). A phase 1 trial of e7974 administered on day 1 of a 21-day cycle in patients with advanced solid tumors. Cancer, 118(17), 4262-4270.

Rojas, V., Rivas, L., Cárdenas, C., \& Guzmán, F. (2020). Cyanobacteria and eukaryotic microalgae as emerging sources of antibacterial peptides. Molecules, 25(24), 5804

Román, J. J. M., Campo, M. D. C., Villar, J., Paolini, F., Curzio, G., Venuti, A., Jara, L., Ferreira, J., Murgas, P., Lladser, A., Manubens, A., \& Becker, M. I. (2019). Immunotherapeutic potential of mollusk hemocyanins in combination with hu- 
man vaccine adjuvants in murine models of oral cancer. Journal of Immunology Research, 2, 1-19.

Rosa, G.-P., Tavares, W.-R., Sousa, P. M. C., Pagès A. K., Seca, A. M. L., \& Pinto, D. C. G. A. (2019). Seaweed secondary metabolites with beneficial health effects: An overview of successes in in vivo studies and clinical trials. Marine Drugs, 18(1), 8

Roth, P., Mason, W. P., Richardson, P. G., \& Weller, M. (2020). Proteasome inhibition for the treatment of glioblastoma. Expert Opinion on Investigational Drugs, 29(10), 1133-1141.

Ruiz-Torres, V., Encinar, J. A., Herranz-López, M., Pérez-Sánchez, A., Galiano, V., Barrajón-Catalán, E., \& Vicente, M. (2017). An updated review on marine anticancer compounds: The use of virtual screening for the discovery of smallmolecule cancer drugs. Molecules, 22(7), 1037.

Sahlmann, C.-O., Homayounfar, K., Niessner, M., Dyczkowski, J., Conradi, L.-C., Braulke, F., Meller, B., Beibarth, T., Ghadimi, B. M., Meller, J., Goldenberg, D. M., \& Liersch, T. (2017). Repeated adjuvant anti-CEA radioimmunotherapy after resection of colorectal liver metastases: safety, feasibility, and long-term efficacy results of a prospective phase 2 study. Cancer, 123(4), 638-649.

Salehi, B., Selamoglu, Z., Mileski, K. S., Pezzani, R., Redaelli, M., Cho, W. C., Kobarfard, F., Rajabi, S., Martorell, M., Kumar, P., Martins, N, Santra, T. S., \& Sharifi-Rad, J. (2019). Liposomal cytarabine as cancer therapy: From chemistry to medicine. Biomolecules, 9(12), 773.

Sang, V. T., Dat, T. T. H., Vinh, L. V., Cuong, L. C. V., Oanh, P. T. T., Ha, H., Kim, Y. H., Anh, H. L. T., \& Yang, S. Y. (2019). Coral and coral-associated microorganisms: A prolific source of potential bioactive natural products. Marine Drugs, 17(8), 468.

Sansone, C., Bruno, A., Piscitelli, C., Baci, D., Fontana, A., Brunet, C., Noonan, D. N., \& Albini, A. (2021). Natural compounds of marine origin as inducers of immunogenic cell death (ICD): Potential role for cancer interception and therapy. Cells, 10(2), 231.

Santos, J. D. S., Vitorino, I., De la Cruz, M., Díaz, C., Cautain, B., Annang, F., PérezMoreno, G., Gonzalez, I., Tormo, J. R., Martin, J., Vicente, M. F., \& Lage, O. (2020). Diketopiperazines and other bioactive compounds from bacterial symbionts of marine sponges. Antonie Van Leeuwenhoek, 113(7), 875-887.

Savinkova, A. V., Zhidkova, E. M., Tilova, L. R., Lavrova, M. D., Lylova, E. S., Kuzin, K. A., Portjannikova, A. J., Maksimova, V. P., Holodova, A. V., Vlasova, O. A., Fetisov, T. I., Kirsanov, K. I., Belickij, G. A., Jakubovskaja, M. G., \& Lesovaja, E. A. (2018). Varianty i perspektivy pereprofilirovanija lekarstvennyh preparatov dlja ispol'zovanija $\mathrm{v}$ terapii onkologicheskih zabolevanij [Options and prospects for repurposing drugs for use in the treatment of oncological diseases]. Sibirskij Onkologicheskij Zhumal, 17(3), 77-87 (in Russian).

Shah, S. A. A., Akhter, N., Auckloo, B. N., Khan, I., Lu, Y., Wang, K., Wu, B., \& Guo, Y.-W. (2017). Structural diversity, biological properties and applications of natural products from cyanobacteria. A review. Marine Drugs, 15(11), 354.

Shapiro, G. I., Vaishampayan, G. I., LoRussio, P., Barton, J., Hua, S., Reich, S. D., Shazer, R., Taylor, C. T., Xuan D., \& Borghaei, H. (2017). First-in-human trial of an anti-5T4 antibody-monomethylauristatin conjugate, PF-06263507, in patients with advanced solid tumors. Investigational New Drugs, 35(3), 315-323.

Sheng, X., Yan, X., Wang, L., Shi, Y., Yao, X., Luo, H., Shi, B., Liu, J., He, Z., Yu, G., Ying, J., Han, W., Hu, C., Ling, Y., Chi, Z., Cui, C., Si, L., Fang, J., Zhou, A., \& Guo, J. (2021). Open-label, multicenter, phase II study of RC48-ADC, a HER2-targeting antibody-drug conjugate, in patients with locally advanced or metastatic urothelial carcinoma. Clinical Cancer Research, 27(1), 43-51.

Sherman, D. J., \& Li, J. (2020). Proteasome inhibitors: Harnessing proteostasis to combat disease. Molecules, 25(3), 671.

Shilabin, A. G., \& Hamann, M. T. (2011). In vitro and in vivo evaluation of select kahalalide $\mathrm{F}$ analogs with antitumor and antifungal activities. Bioorganic and Medicinal Chemistry, 19(22), 6628-6632.

Singh, A. V., Bandi, M., Raje, N., Richardson, P., Palladino, M. A., Chauhan, D., \& Anderson, K. C. (2011). A novel vascular disrupting agent plinabulin triggers JNK-mediated apoptosis and inhibits angiogenesis in multiple myeloma cells. Blood, 117(21), 5692-5700.

Singh, I., Lacko, C. S., Zhao, Z., Schmidt, C. E., \& Rinaldi, C. (2020). Preparation and evaluation of microfluidic magnetic alginate microparticles for magnetically templated hydrogels. Journal of Colloid and Interface Science, 561, 647-658.

Skidmore, L., Sakamuri, S., Knudsen, N. A., Hewet, A. G., Milutinovic, S., Barkho, W., Biroc, S. L., Kirtley, J., Marsden, R., Storey, K., Lopez, I., Yu, W., Fang, S.Y., Yao, S., Gu, Y., \& Tian, F. (2020). ARX788, a site-specific anti-HER2 antibody-drug conjugate, demonstrates potent and selective activity in HER2-low and T-DM1-resistant breast and gastric cancers. Molecular Cancer Therapeutics, 19(9), 1833-1843.

Smith, T. E., Pond, C. D., Pierce, E., Harmer, Z. P., Kwan, J., Zachariah, M. M., Harper, M. K., Wyche, T. P., Matainaho, T. K., Bugni, T. S., Barrows, L. R., Ireland, C. M., \& Schmidt, E. W. (2018). Accessing chemical diversity from the uncultivated symbionts of small marine animals. Nature Chemical Biology, 14(2), 179-185.

Straus, D. J., Długosz-Danecka, M., Alekseev, S., Illés, Á., Picardi, M., Lech-Maranda, E., Feldman, T., Smolewski, P., Savage, K. J., Bartlett, N. L., Walewski, J.,
Ramchandren, R., Zinzani, P. L., Hutchings, M., Connors, J. M., Radford, J., Munoz, J., Kim, W. S., Advani, R., Ansell, S. M., Younes, A., Miao, H., Liu, R., Fenton, K., Forero-Torres, A., \& Gallamini, A. (2020). Brentuximab vedotin with chemotherapy for stage III/IV classical Hodgkin lymphoma: 3-year update of the ECHELON-1 study. Blood, 135(10), 735-742.

Strickler, J. H., Weekes, C. D., Nemunaitis, J., Ramanathan, R. K., Heist, R. S., Morgensztern, D. M., Angevin, E., Bauer, T. M., Yue, H., Motwani, M., Parikh, A., Reilly, E. B., Afar, D., Naumovski, L., \& Kelly, K. (2018). First-in-human phase I, dose-escalation and expansion study of telisotuzumab vedotin, an antibody-drug conjugate targeting c-met, in patients mith advanced solid tumors. Joumal of Clinical Oncology, 36(33), 3298-3306.

Stuijvenberga, J., Prokschb, P., \& Fritz, G. (2020). Targeting the DNA damage response (DDR) by natural compounds. Bioorganic and Medicinal Chemistry, 28(4), 115279

Subjakova, V., Oravczova, V., \& Hianik, T. (2021). Polymer nanoparticles and nanomotors modified by DNA/RNA aptamers and antibodies in targeted therapy of cancer. Polymers, 13(3), 341.

Summer, K., Browne, J., Liu, L., \& Benkendorff, K. (2020). Molluscan compounds provide drug leads for the treatment and prevention of respiratory disease. Marine Drugs, 18(11), 570.

Taglialatela-Scafati, O. (2021). New hopes for drugs against COVID-19 come from the sea. Marine Drugs, 19(2), 104.

Takahashi, R., Mabuchi, S., Kawano, M., Sasano, T., Matsumoto, Y., Kuroda, H., Kozasa, K., Hashimoto, K., Sawada, K., \& Kimura, T. (2016). Preclinical investigations of PM01183 (lurbinectedin) as a single agent or in combination with other anticancer agents for clear cell carcinoma of the ovary. Plos One, 11(3), 0151050.

Tan, Y. Y., Yap, P. K., Lim, G. L. X., Mehta, M., Chanc, Y., Ng, S. W., Kapoor, D. N., Negi, P., Anand, K., Singh, S. K., Jha, N. K., Lim, L. C., Madheswaran, T., Satija, S., Gupta, G., Dua, K., \& Chellappan, D. K. (2020). Perspectives and advancements in the design of nanomaterials for targeted cancer theranostics. Chemico-Biological Interactions, 329, 109221.

Tannir, N. M., Forero-Torres, A., Ramchandren, R., Pal, S. K., Ansell, S. M., Infante, J. R., De Vos, S., Hamlin, P. A., Kim, S. K., Whiting, N. C., Gartner, E. M., Zhao, B., \& Thompson, J. A. (2014). Phase I dose-escalation study of SGN-75 in patients with CD70-positive relapsed/refractory non-Hodgkin lymphoma or metastatic renal cell carcinoma. Investigational New Drugs, 32(6), 1246-1257.

Teplinsky, E., \& Herzog, T. J. (2017). The efficacy of trabectedin in treating ovarian cancer. Expert Opinion on Pharmacotherapy, 18(3), 313-323.

Tian, Y., Qiu, X., Wang, C., Zhao, J., Jiang, X, Niu, W., Huang, J., \& Zhang, F. (2021). Cancer associates with risk and severe events of COVID-19: A systematic review and meta-analysis. International Journal of Cancer, 148(2), 363-374.

Tjuljandin, S. A., \& Zhukov, N. V. (2018). Pravda o rossijskoj onkologii: Problemy i vozmozhnye reshenija [The truth about Russian oncology: Problems and possible solutions]. Rossijskoe Obshhestvo Klinicheskoj Onkologii, Moscow (in Russian).

Tolaney, S. M., Barroso-Sousa, R., Keenan, T., Li, T., Trippa, L., Vaz-Luis, I., Wulf, G., Spring, L., Sinclair, N. F., Andrews, C., Pittenger, J., Richardson, E. T., Dillon, D., Lin, N. U., Overmoyer, B., Partridge, A. H., Van Allen, E., Mittendorf, E. A., Winer, E. P., \& Krop, I. E. (2020). Effect of eribulin with or without pembrolizumab on progression-free survival for patients with hormone receptor-positive, ERBB2-negative metastatic breast cancer: A randomized clinical trial. JAMA Oncology, 6(10), 1598-1605.

Tolcher, A. W. (2020). The evolution of antibody-drug conjugates: A positive inflexion point. American Society of Clinical Oncology Educational Book, 40, 127-134.

Tonra, J. R., Lloyd, G. K., Mohanlal, R., \& Huang, L. (2020). Plinabulin ameliorates neutropenia induced by multiple chemotherapies through a mechanism distinct from G-CSF therapies. Cancer Chemotherapy and Pharmacology, 85(2), 461-468.

Tsai, H.-L., Tai, C.-J., Huang, C.-W., Chang, F.-R., \& Wang, J.-Y. (2017). Efficacy of low-molecular-weight fucoidan as a supplemental therapy in metastatic colorectal cancer patients: A double-blind randomized controlled trial. Marine Drugs, 15(4), 122.

Tzogani, K., Penttilä, K., Lähteenvuo, J., Lapveteläinen, T., Anglada, L., Prieto, C., Garcia-Ochoa, B., Enzmann, H., Gisselbrecht, C., Delgado, J., \& Pignatti, F. (2021). Ema review of belantamab mafodotin (blenrep) for the treatment of adult patients with relapsed/refractory multiple myeloma. Oncologist, 26(1), 70-76.

Van Andel, L., Rosing, H., Schellens, J. H. M., \& Beijnen, J. H. (2018). Review of chromatographic bioanalytical assays for the quantitative determination of marine-derived drugs for cancer treatment. Marine Drugs, 16(7), 246.

Van Den Bent, M., Eoli, M., Sepulveda, J. M., Smits, M., Walenkamp, A., Frenel, J. S., Franceschi, E., Clement, P. M., Chinot, O., De Vos F., Whenham, N., Sanghera, P., Weller, M., Dubbink, H. J., French, P., Looman, J., Dey, J., Krause, S., Ansell, P., Nuyens, S., Spruyt, M., Brilhante, J., Coens, C., Gorlia, T., \& Golfinopoulos, V. (2020). Intellance 2/EORTC 1410 randomized phase II study of Depatux-M alone and with temozolomide vs temozolomide or lomustine in recurrent EGFR amplified glioblastoma. Neuro-Oncology, 22(5), 684-693. 
Van Stuijvenberg, J., Proksch, P., \& Fritz, G. (2020). Targeting the DNA damage response (DDR) by natural compounds. Bioorganic and Medicinal Chemistry, 28(4), 115279.

Vijayakumar, S., \& Menakha, M. (2015). Pharmaceutical applications of cyanobacteria. Joumal of Acute Medicine, 5(1), 15-23.

Wang, L., Dong, C., Li, X., Han, W., \& Xiulan, S. (2017). Anticancer potential of bioactive peptides from animal sources (review). Oncology Reports, 38(2), $637-651$.

Wang, P., Kankala, R. K. K., Chen, B., Long, R., Cai, D., Liu, Y., \& Wang, S. (2019). Poly-allylamine hydrochloride and fucoidan-based self-assembled polyelectrolyte complex nanoparticles for cancer therapeutics. Journal of Biomedical Materials Research, 107(2), 339-347.

Wang, Q., Liu, S., Yang, F., Gan, L., Yang, X., \& Yang, Y. (2017). Magnetic alginate microspheres detected by MRI fabricated using microfluidic technique and release behavior of encapsulated dual drugs. International Joumal of Nanomedicine, 12, 4335-4347.

Wang, X., Ma, D., Olson, W. C., \& Heston, W. D. W. (2011). In vitro and in vivo responses of advanced prostate tumors to PSMA ADC, an auristatin-conjugated antibody to prostate-specific membrane antigen. Molecular Cancer Therapeutics, 10(9), 1728-1739.

Washah, H., Agoni, C., Olotu, F. A., Munsamy, G., \& Soliman, M. E. S. (2020). Tweaking $\alpha$-galactoceramides: Probing the dynamical mechanisms of improved recognition for invariant natural killer T-cell receptor in cancer immunotherapeutics. Current Pharmaceutical Biotechnology, 21(13), 1354-1367.

Watters, D. J. (2018). Ascidian toxins with potential for drug development. Marine Drugs, 16(5), 162.

Weekes, C. D., Lamberts, L. E., \& Borad, M. J. (2016). Phase I study of DMOT4039A, an antibody-drug conjugate targeting mesothelin, in patients with unresectable pancreatic or platinum-resistant ovarian cancer. Large Molecule Therapeutics, 15(3), 439-47.

White, K. M., Rosales, R., Yildiz, S., Kehrer, T., Miorin, L., Moreno, E., Jangra, S., Uccellini, M. B., Rathnasinghe, R., Coughlan, L., Martinez-Romero, C., Batra, L., Rojc, A., Bouhaddou, M., Fabius, J. M., Obernier, K., Dejosez, M., Guillén, M. J., Losada, A., Avilés, P., Schotsaert, M., Zwaka, T., Vignuzzi, M., Shokat K. M., Krogan, N. J., \& García-Sastre, A. G. (2021). Plitidepsin has potent preclinical efficacy against SARS-CoV-2 by targeting the host protein eEF1A. Science, 371(6532), 926-931.

Wimmers, F., De Haas, N., Scholzen, A., Schreibelt, G., Simonetti, E., Eleveld, E., Brouwers, H. M. L. M., Beldhuis-Valkis, M., Joosten, I., De Jonge, M. I., Gerritsen, W. R., De Vries, I. J. M., Diavatopoulos, D. A., \& Jacobs, J. F. M. (2017). Monitoring of dynamic changes in Keyhole Limpet Hemocyanin (KLH)-specific B cells in KLH-vaccinated cancer patients. Scientific Reports, 7, 43486.

Wolska-Washer, A., \& Robak, T. (2019). Safety and tolerability of antibody-drug conjugates in cancer. Drug Safety, 42(2), 295-314.

Won, Y. C., Kim, H. J., \& Hun, L. S. (2019). Therapeutic application of diverse marinederived natural products in cancer therapy. Anticancer Research, 39(10), 52615284.

Xi, X. D., \& Dong, B. (2019). Origins and bioactivities of natural compounds derived from marine ascidians and their symbionts. Marine Drugs, 17(12), 670.

Xing, H., Tong, M., Jiang, N., Zhang, X., Hu, H., Pan, H., \& Li, D. (2017). Antitumor bioactive peptides isolated from marine organisms. Clinical and Experimental Pharmacology and Physiology, 44(11), 1077-1082.
Xu, Y., Kersten, R. D., Nam, S. J., Lu, L., Al-Suwailem, A. M., Zheng, H., Fenical, W., Dorrestein, P. C., Moore, B., \& Qian, P.-Y. (2012). Bacterial biosynthesis and maturation of the didemnin anti-cancer agents. Journal of the American Chemical Society, 134(20), 8625-8632.

Yang, B., Huang, J., Zhou, X., Lin, X., Liu, J., Liao, S., Wang, J., Liu, F.-A., Huaming, T., \& Liu, Y. (2018). The fungal metabolites with potential antiplasmodial activity. Current Medicinal Chemistry, 25(31), 3796-3825.

Yang, K., Chen, B., Gianolio, D. A., Stefano, J. E., Busch, M., Manning, C., Alving, K., Gregory, R. C., Brondyk, W. H., Miller, R. J., \& Dhal, P. K. (2019). Convergent synthesis of hydrophilic monomethyl dolastatin 10 based drug linkers for antibody-drug conjugation. Organic and Biomolecular Chemistry, 17(35), 8115-8124.

Ye, C., Tan, Y. F., Wong, Y. S., Liew, M. W. L., \& Venkatraman, S. (2019). Recent advances in chitosan-based carriers for gene delivery. Marine Drugs, 17(6), 381.

Youssef, F. S., Alshammari, E., \& Ashour, M. L. (2021). Bioactive alkaloids from genus Aspergillus: Mechanistic interpretation of their antimicrobial and potential SARS-CoV-2 inhibitory activity using molecular modelling. International Journal of Molecular Sciences, 22(4), 1866.

Youssef, F. S., Ashour, M. L., Singab, A. N. B., \& Wink, M. (2019). A comprehensive review of bioactive peptides from marine fungi and their biological significance. Marine Drugs, 17(10), 559.

Yu, S. F., Lee, D. W., Zheng, B., Del Rosario, G., Leipold, D., Booler, H., Zhong, F., Carrasco-Triguero, M., Hong, K., Yan, P., Rowntree, R. K., Schutten, M. M., Pillow, T., Sadowsky, J. D., Dragovich, P. S., \& Polson, A. G. (2021). An antiCD22-seco-CBI-dimer antibody-drug conjugate (ADC) for the treatment of non-Hodgkin lymphoma that provides a longer duration of response than auristatin-based ADCs in preclinical models. Molecular Cancer Therapeutics, 20(2), 340-346.

Yuan, P., \& Xu, B. (2021). Clinical utility of eribulin mesylate in the treatment of breast cancer: A chinese prspective. Breast Cancer, 13, 135-150.

Yurkovetskiy, A. V., Bodyak, N. D., Yin, M., Thomas, J. D., Clardy, S. M., Conlon, P. R., Stevenson, C. A., Uttard, A., Qin, L. L., Gumerov, D. R., Ter-Ovanesyan, E., Bu, C., Johnson, A. J., Gurijala, V. R., McGillicuddy, D., DeVit, M. J., Poling, L. P., Protopopova, M., Xu, L., Zhang, Q., Park, P. U., Bergstrom, D. A., \& Lowinger, T. B. (2021). Dolaflexin: A novel antibody-drug conjugate platform featuring high drug loading and a controlled bystander effect. Molecular Cancer Therapeutics, 20(5), 885-895.

Zahran, E. M., Albohy, A., Khalil, A., Ibrahim, A. H., Ahmed, H. A., El-Hossary, E. M., Bringmann, G., \& Abdelmohsen, U. R. (2020). Bioactivity potential of marine natural products from scleractinia-associated microbes and in silico antiSARS-COV-2 evaluation. Marine Drugs, 18(12), 645.

Zhang, H., Zhao, Z., \& Wang, H. (2017). Cytotoxic natural products from marine sponge-derived microorganisms. Marine Drugs, 15(3), 68.

Zhang, Q.-T., Liu, Z.-D., Wang, Z., \& Wang, T. (2021). Recent advances in small peptides of marine origin in cancer therapy. Marine Drugs, 19(2), 115.

Zhao, P., Zhang, Y., Li, W., Jeanty, C., Xiang, G., \& Dong, Y. (2020). Recent advances of antibody drug conjugates for clinical applications. Acta Pharm Sin B, 10(9), 1589-1600.

Zhong, H., Gao, X., Cheng, C., Liu, C., Wang, Q., \& Han, X. (2020). The structural characteristics of seaweed polysaccharides and their application in gel drug delivery systems. Marine Drugs, 18(12), 658. 